\title{
Resonance Vibration Control for AMB Flexible Rotor System Based on $\mu$-Synthesis Controller
}

\author{
Shaolin Ran, ${ }^{1}$ Yefa Hu, ${ }^{1,2}$ Huachun Wu $\mathbb{D}^{1,2}$ and Xin Cheng ${ }^{1,2}$ \\ ${ }^{1}$ School of Mechanical and Electronic Engineering, Wuhan University of Technology, Wuhan 430070, China \\ ${ }^{2}$ Hubei Provincial Engineering Technology Research Center for Magnetic Suspension, Wuhan 430070, China \\ Correspondence should be addressed to Huachun Wu; whcwhut@whut.edu.cn
}

Received 21 September 2018; Revised 22 November 2018; Accepted 3 December 2018; Published 19 December 2018

Academic Editor: Libor Pekař

Copyright (c) 2018 Shaolin Ran et al. This is an open access article distributed under the Creative Commons Attribution License, which permits unrestricted use, distribution, and reproduction in any medium, provided the original work is properly cited.

\begin{abstract}
The resonance vibration control of flexible rotor supported on active magnetic bearings (AMB) is a challenging issue in the industrial applications. This work addresses the application of robust control method to the resonance vibration control for AMB flexible rotor while passing through the critical speed. This model-based method shows great superiority to handling flexible mode vibration, which can guarantee robust stability and performance when encountering modal perturbation. First, the designed flexible rotorAMB test rig is briefly introduced. Then the system modeling is described in detail including flexible rotor, power amplifier, displacement sensors and magnetic actuator and rotordynamics are analyzed. Model validation is carried out by sine sweeping test. Finally, the $\mu$-synthesis controller is designed. The simulation and experimental results indicate that the designed $\mu$-synthesis controller, which shows great robustness to modal perturbation, can effectively suppress the resonance vibration of flexible rotor and achieve supercritical operation.
\end{abstract}

\section{Introduction}

Active magnetic bearing (AMB) is a typically mechatronical bearing which can make the rotor suspend stably through active feedback control. It has many characters such as contactless, no-friction, and no-lubrication [1]. Benefiting from these characters, AMBs enable the machines with a very high rotational speed and has been widely used in compressors [2], motors [3], and flywheel system [4]. High rotational speed brings about many benefits such as high energy efficiency and compactness for the energy-saving and emission-reduction [5]. In these cases, because of structure limitation, the high speed machines have to operate above the bending critical speeds [6]. However, the flexible rotor encounters resonance before reaching its rated speed. Resonance vibration can cause serious damage to the device. Hence, the resonance vibration suppression of flexible rotor becomes a challenging issue.

Compared with general control of flexible space structure, such as flight control [7] and robotic [8] and hard disk control [9], control of rotor's flexible mode in AMB system can be a more challenging issue, because the $\mathrm{AMB}$ is inherently open-loop unstable system, which has fundamental limitations for closed-loop stability. Subject to gyroscopic effect and rotor internal damping, rotor dynamic is inherently speed dependent, which complicates the controller design [10]. Meanwhile, due to the nonlinear material properties in laminations, shrink fits, assembling and manufacture error, the rotor modal perturbation should be considered during controller design [11]. Therefore, the resonance vibration control of flexible rotor has been the focus of much research over the past decades.

PID is the most widely used control method in the industry for its simple structure. So, researchers resort to PID controller integrated with different general second order filters to achieve good resonance vibration control effect for $\mathrm{AMB}$ flexible rotor [12-14]. In these literatures, the optimal damping and optimal phase angle are derived in detail based on the plant phase information nearby the rotor bending mode, then phase lead filter and notch filter are connected in series after the PID controller based on the optimal phase angle. The added filters extend the application of PID for flexile mode control, which also complicate the control system nevertheless. This standard control method does not allow for 


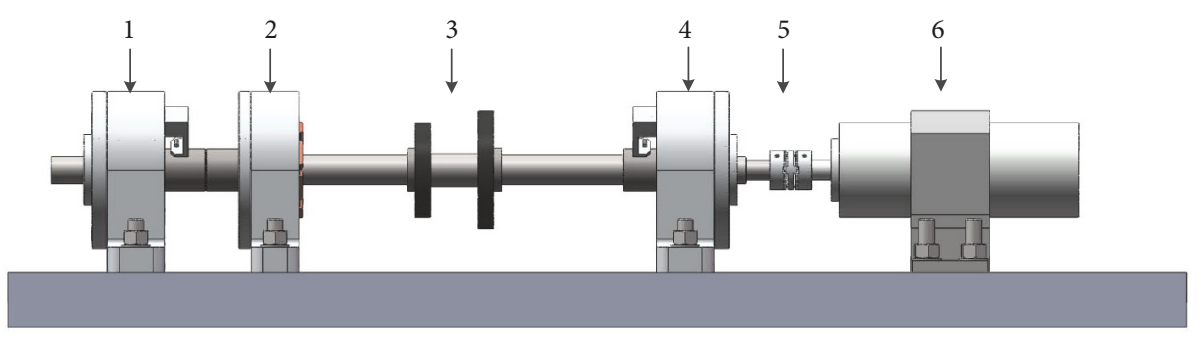

(1) Radial AMB (2) Exciting AMB (3) Rotor (4) Radial AMB (5) Flexible coupling (6) Motor

FIgURE 1: Flexible rotor test rig.

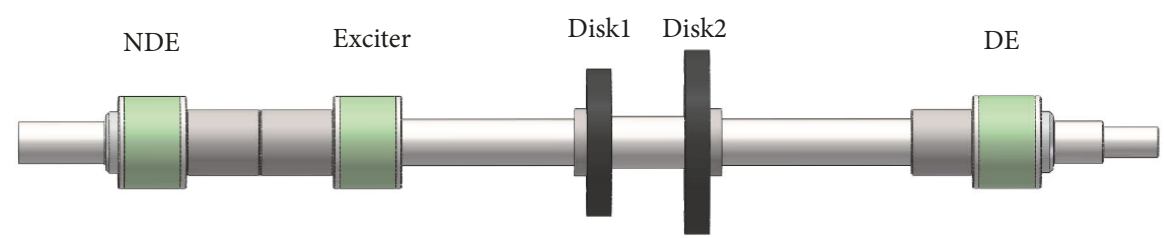

FIGURE 2: Flexible rotor.

taking into account all factors that can influence the static and dynamic quality of controlled process [15].

The robust control method, such as Ho and $\mu$-synthesis, can take direct account of system uncertainties in the controller design procedure for actively reducing vibration of rotor systems [16]. The early research focuses on the constructions and solving procedure of the robust control problem [17-19] and mainly focuses on the rigid rotor [20-22]. Then, many scholars turn to flexible rotor robust control based on finite element method modeling. Ho control method is successfully applied to the AMB flexible rotor system and the analytical and experimental results show the effectiveness of the Ho control [23, 24]. However, the Ho control is based on unstructured uncertainties. When encountered with parametric uncertainties, the synthesized controller may be conservative. Compared to Ho control, the $\mu$-synthesis can address explicitly the parametric uncertainty in system and specify the performance specification to reduce the controller conservatism. Jinxiang Zhou et al. applied $\mu$ synthesis control method to the AMB system for dealing with parameters uncertainty of linear electromagnetic force model and weighting functions are introduced based on the objectives of AMBs performance. The experimental results showed desired performance of the AMB with $\mu$ controller [25]. In [26], rotor instability phenomenon affected by aerodynamic cross-coupled stiffness forces is studied by designing a rotor test rig with magnetic bearings and a $\mu$-synthesis controller was designed. The research results indicate that $\mu$-synthesis controller can achieve better control effect on the stability of rotor 1st bending mode under cross-coupled stiffness excitation. Although $\mu$-synthesis controller can promote the performance of AMB system on the rotor resonance vibration, some degree of system modeling accuracy must be guaranteed.

In this paper, a transparent procedure of control oriented rotor-AMB system modeling and $\mu$-synthesis controller design is presented. Both the simulation and experimental results demonstrate that the designed controller has better rotor resonance vibration suppression performance and high robustness to system uncertainty to make the flexible rotor exceed the first bending critical speed smoothly.

\section{The Flexible Rotor-AMB Test Rig}

The purpose of the flexible rotor-AMB rest rig is to study rotor vibration control and supercritical operation as shown in Figure 1.

Two radial magnetic bearings are placed at both ends of the rotor. An exciter AMB in quarter spans of the rotor is used to exert different excitation on the rotor. The rotor is connected with a $4 \mathrm{~kW}$ motor through a flexible coupling. Four eddy current displacement sensors are installed besides two bearings respectively to measure the displacement of the rotor in $x$ and $y$ directions. When the control is off, the rotor is down on the auxiliary bearings.

The rotor is $0.93 \mathrm{~m}$ long and weighs around $18.14 \mathrm{~kg}$ with three laminated steel journals. Two radial support AMBs are at the nondriven end (NDE) and driven end (DE). Only the NDE and DE support AMBs are utilized for control. The sketch of the flexible rotor in this work is shown in Figure 2.

\section{Modeling of AMB System}

$\mu$-Synthesis is fundamentally a model-based control method requiring a detailed theoretical model of the system. Therefore, the modeling of the AMB system is very important. The AMB system includes controller, power amplifier, rotor and stator, and displacement sensors. A typical control diagram of the magnetic bearing system is shown in Figure 3, which shows the signal exchange of each electromechanical component. In order to get a model-based controller with better performance, a relatively accurate system model should be obtained. 


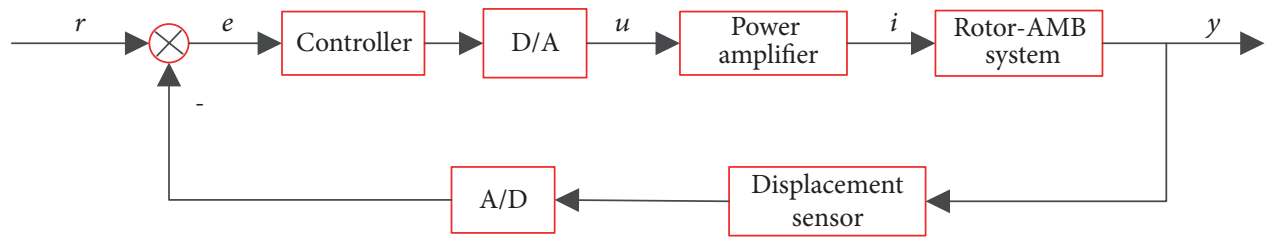

FIGURE 3: Typical control schematic diagram of magnetic bearing system.

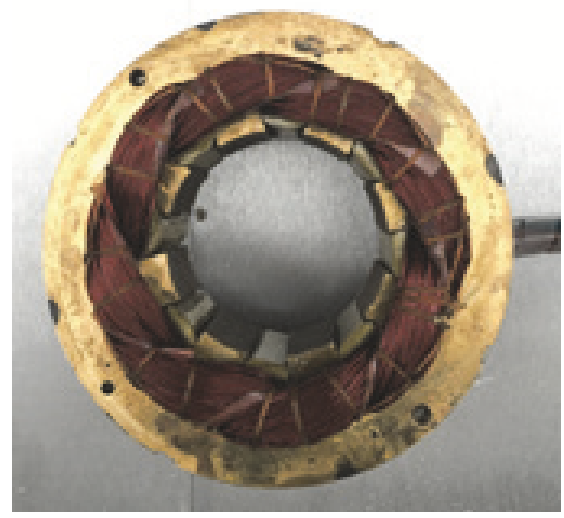

(a)

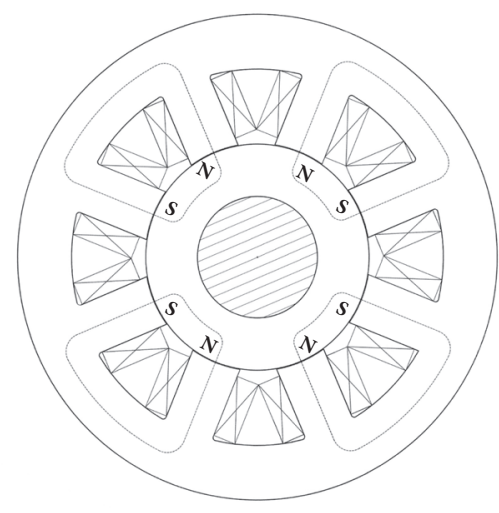

(b)

FIGURE 4: Structure of stator.

3.1. Modeling of the AMB. The support AMB stators are 8pole heteropolar bearings with laminated silicon-iron. The orientation of the quarter magnet is offset $45^{\circ}$ from vertical, allowing the rotor weight to be distributed equally between the two control channels. This ensures the $x$ and $y$ dynamic will be nearly identical. The stator is shown in Figure 4 .

Affected by current and gap, the magnetic force is inherently nonlinear. In order to simplify the control design, an approximate linear model can be as follows:

$$
F=k_{i} i-k_{x} x
$$

where $k_{i}$ is current stiffness and $k_{x}$ is negative displacement stiffness. $k_{i}$ and $k_{x}$ only depend on the physical characteristics.

There will always be discrepancy between the actual bearing performance and analytical model due to the errors in the manufacture and linearization process. Therefore, in order to obtain the exact actuator performance, the current stiffness and displacement stiffness are determined by test data which contribute to the system modeling. When the rotor is suspended by PID controller, in $x$ direction of the rotor, the control current will be adjusted to keep the rotor at the equilibrium position under a force exerted by the pull meter. In this way, a series of forces and currents data will be recorded, and the relationship between forces and currents is obtained as shown in Figure 5.

Then, when the pull meter is removed and the rotor is suspended at different positions in the $x$ direction, a series of displacements and currents data is obtained, and their relationship is shown in Figure 6.

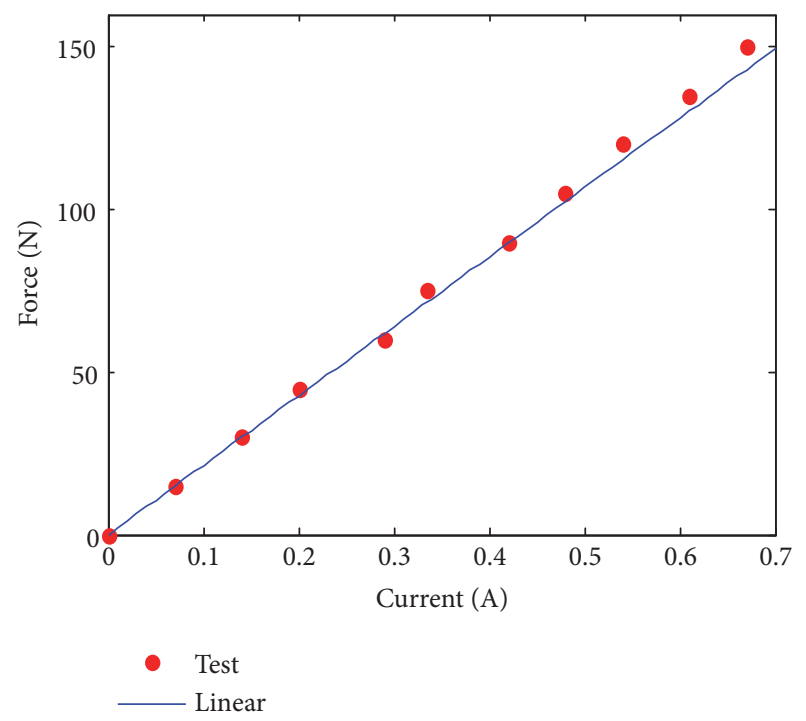

FIGURE 5: Relationship of forces and currents.

The slope of the force-current curve is the current stiffness $k_{i}$, which can be obtained by linear fitting. The displacement stiffness $k_{x}$ can be derived as [27]

$$
k_{x}=-k_{i} \frac{d i}{d x}
$$

where $d i / d x$ is the slope of the current-displacement curve, which can also be obtained linear fitting.

Finally, $k_{i}=213.5 \mathrm{~N} / \mathrm{A}, k_{x}=-1110.2 \mathrm{~N} / \mathrm{mm}$. 


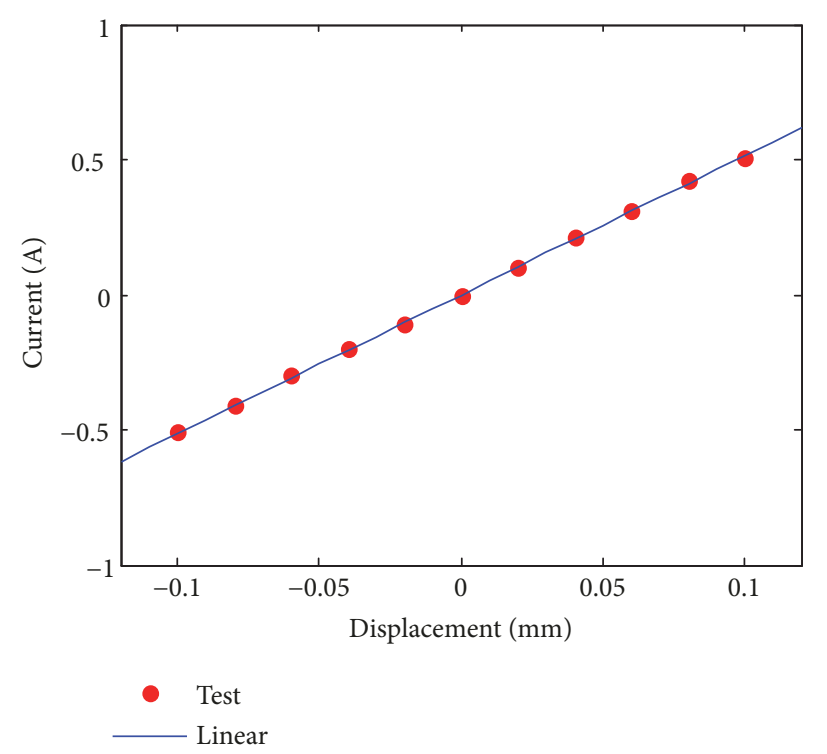

FIGURE 6: Relationship of currents and displacements.

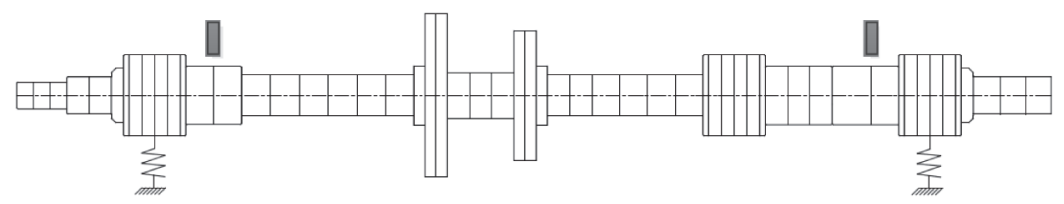

FIgURE 7: The FE model of the rotor.

3.2. Modeling and Analysis of Flexible Rotor. In this work, the finite element (FE) method is employed to model the flexible rotor. As shown in Figure 7, the rotor is divided into 56 units with 57 nodes. Based on the Timoshenko beam theory, the motion equation of the rotor is as follows:

$$
\begin{aligned}
M \ddot{u}+(D+\Omega J) \dot{u}+K u & =B_{1} F_{\text {mag }}+B_{2} f \\
y & =C u
\end{aligned}
$$

where $M, D, J$, and $K$ denote the mass matrix, internal shaft damping matrix, gyroscopic matrix, and stiffness matrix, respectively. They are all symmetric and positive definite except for that the gyroscopic matrix is skew-symmetric; $u$ represents translations along the $x$ and $y$ axes and angular displacement about the $y$ and $x$ axes. The $4 \times 1$ vector $F_{\text {mag }}$ denotes the electromagnetic force. $B_{1}$ is the distribution matrix of magnetic force; $f$ is external force acting on the rotor (mainly are unbalanced forces). Details regarding the FE modeling procedures for rotors are available in [28].

Based on the developed FE model, the lumped-mass method is employed to obtain the modal frequencies of the rotor in the free-free state, which are $152.56 \mathrm{~Hz}, 423.59 \mathrm{~Hz}$, 866.65 Hz, $1312.34 \mathrm{~Hz}$, and $1752.92 \mathrm{~Hz}$, respectively. However, the shrink-fitting silicon steel sheet and sleeves on the rotor will undoubtedly promote the bending stiffness, which has an important influence on the rotor modal frequency. Therefore, it is necessary to update the theoretical FE model of the rotor according to the experimental modal data of the rotor.
Experimental modal analysis is an effective method for updating rotor model. In order to get the real modal parameters of the rotor, a LMS vibration and noise testing system is employed to conduct modal test. The rotor is hung with flexible string to emulate the free-free state, and finally the first five bending modal frequencies of the rotor are obtained, as shown in Figure 8.

Generally, the physical parameters of the rotor, such as material density, mass, and geometry, are constant. However, in the FE modeling process, the elastic modulus of elements becomes an engineering assumption due to influence of the shrink-fitting components. Thus, elastic modulus of the rotor with shrink-fitting components can be taken as a manual parameter, and the FE model can be made approximate to the real rotor characteristics by adjusting the parameters. After lots of trial and error, the updated model modal frequencies are approximate to the modal test data as listed in Table 1.

An important graphic tool for rotordynamic analysis is the undamped free-free mode shape plot, which is shown in Figure 9.

The undamped modal shape can reflect the behavior of the rotor at the sensors and actuators position which can make it clear to us whether the distribution of sensors and actuators is reasonable. Figure 9 shows that the first bending mode of the rotor has good controllability and observability from the control perspective.

Analyzing the rotor critical speed under different support stiffness is very important for predicting the resonance 
TABLE 1: Rotor modal frequency.

\begin{tabular}{lcccc}
\hline & Original value $(\mathrm{Hz})$ & Experimental value $(\mathrm{Hz})$ & Reconciled value $(\mathrm{Hz})$ & Percentage Error $(\%)$ \\
\hline 1st bending & 152.56 & 161.51 & 161.54 & 0.0186 \\
2nd bending & 423.59 & 476.53 & 475.61 & 0.1931 \\
3rd bending & 866.65 & 958.80 & 958.44 & 0.0375 \\
4th bending & 1312.34 & 1528.23 & 1527.82 & 0.0268 \\
5th bending & 1752.92 & 1923.69 & 1935.01 & 0.5885 \\
\hline
\end{tabular}

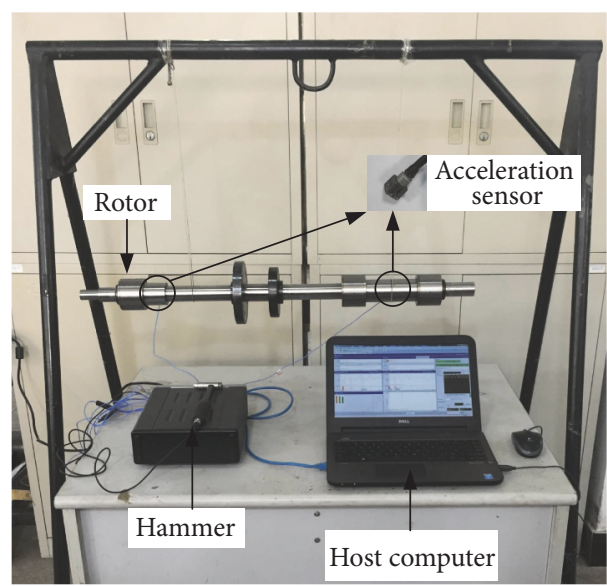

(a) Modal impact test

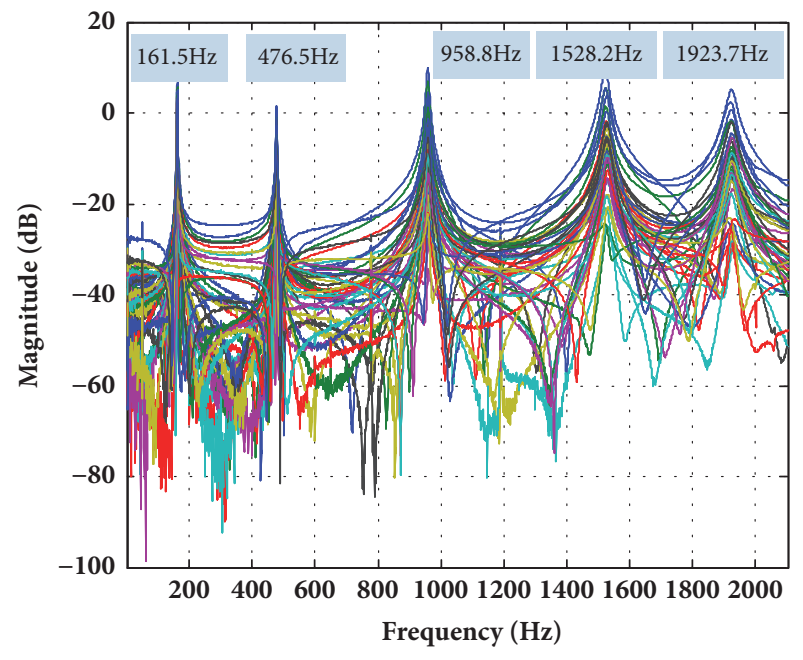

(b) Modal test results

Figure 8: Modal test.

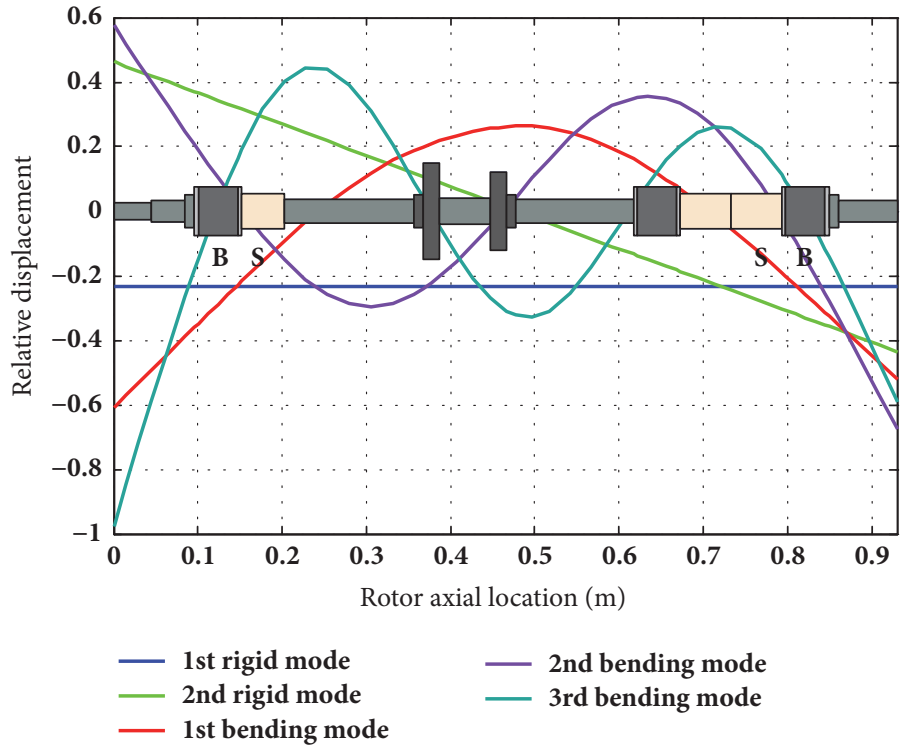

FIGURE 9: Undamped modal shape of the rotor.

interval. Compared with ball bearing, the support stiffness of AMB is quite low. Thus, rotor critical speed is sensitive to support stiffness changes. This phenomenon is very important in the controller design because the controller should be robust to the rotor frequency perturbation. The rotor critical speed map is provided in Figure 10. It is indicated that there is a slight critical speed perturbation. This uncertainty must be considered in the controller design.
The order of updated rotor model obtained through modal test is very large, which will make controller design difficult. Therefore, a reduced order rotor model is needed. Generally, modal truncation is used to reduce the rotor model order. In this work, the first two bending modals of the rotor are preserved.

The gyroscopic effect can be ignored, because the axial/ radial inertia ratio of the flexible rotor is always small. An 


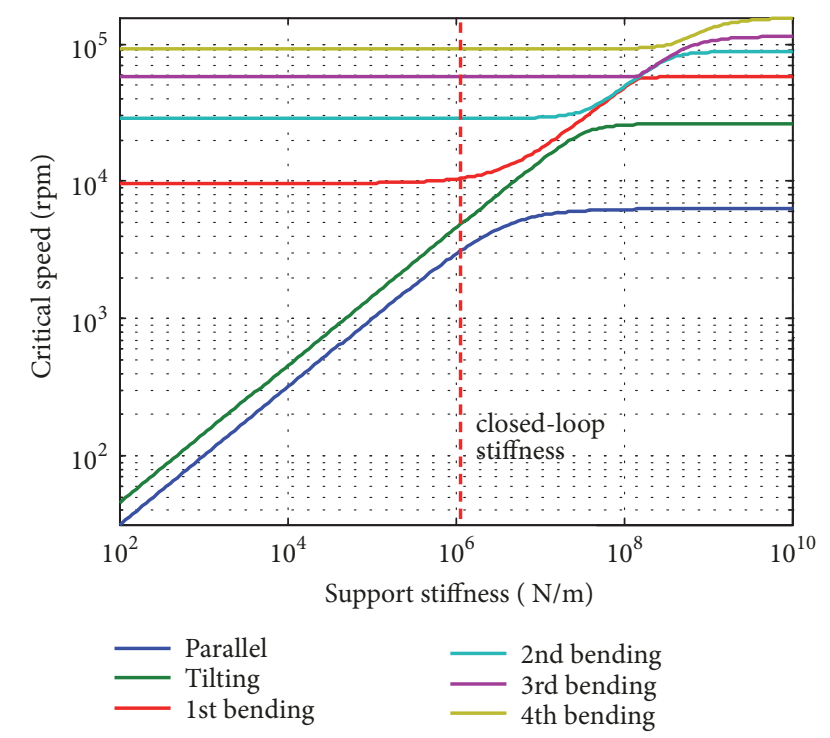

FIgURE 10: Relationship between critical speed and support stiffness.

axisymmetric rotor can be decoupled on the two vertical planes. If only the transverse vibration characteristics of a single plane ( $x-z$ plane) of the rotor are considered, its motion equations can be simplified as follows:

$$
\begin{aligned}
M_{x} \ddot{u}_{x}+D_{x} \dot{u}_{x}+K_{x} u_{x} & =B_{1 x} F_{\text {magx }} \\
y & =C_{x} u_{x}
\end{aligned}
$$

The equation can be transferred to modal space through the modal transfer matrix $\Phi$. Usually, the internal damping of the rotor is small. Thus, to better reflect the behavior of physical rotor, low levels of modal damping ( $0.3 \%)$ were added. The mass normalized motion equation can be obtained.

$$
\begin{aligned}
\ddot{\eta}+2 \zeta \Lambda \dot{\eta}+\Lambda^{2} \eta & =\Phi^{T} B_{1 x} F_{\text {mag } x} \\
y & =C_{x} \Phi \eta
\end{aligned}
$$

where $u=\Phi \eta ; \zeta=0.003$.

The rotor model above can be expressed as state-space form.

$$
\begin{aligned}
& \dot{x}_{m}=\left[\begin{array}{cc}
0 & I \\
-\Lambda^{2} & -2 \zeta \Lambda
\end{array}\right] x_{m}+\left[\begin{array}{c}
0 \\
\Phi^{T} B_{1 x}
\end{array}\right] F_{\text {magx }} \\
& y_{m}=\left[\begin{array}{ll}
C_{x} \Phi & 0
\end{array}\right] x_{m}
\end{aligned}
$$

The magnetic force can be assembled to the state-space equation of rotor. Finally, the order-reduced rotor model can be obtained.

$$
\begin{aligned}
& \dot{x}_{m}=\left[\begin{array}{cc}
0 & I \\
-\left(\Lambda^{2}-\Phi^{T} B_{1 x} K_{x} B_{1 x}^{T} \Phi\right) & -2 \zeta \Lambda
\end{array}\right] x_{m} \\
& +\left[\begin{array}{c}
0 \\
\Phi^{T} B_{1 x} K_{i}
\end{array}\right] i_{c} \\
& y_{m}=\left[\begin{array}{ll}
C_{x} \Phi & 0
\end{array}\right] x_{m} \\
& \text { where } K_{x}=\left[\begin{array}{cc}
k_{x} & 0 \\
0 & k_{x}
\end{array}\right], K_{i}=\left[\begin{array}{cc}
k_{i} & 0 \\
0 & k_{i}
\end{array}\right] .
\end{aligned}
$$

3.3. Modeling of Other Electrical Components. Power amplifier is a key component of the closed-loop system which receives control voltage and generates enough current for AMB coils. The power amplifier dynamics is affected by the inductance of $\mathrm{AMB}$ coils and eddy current effects due to rotor motion. The dashed line in Figure 11 indicates the frequency response of power amplifier while the rotor is centered using copper foil. The transfer function fitted to the experimental frequency response is as follows:

$$
G_{a}(s)=K_{a} \frac{\left(T_{a} s+1\right) \omega_{a}^{2}}{\left(s^{2}+2 \xi \omega_{a} s+\omega_{a}^{2}\right)}
$$

where $K_{a}=1, T_{a}=0.00012, \omega_{a}=530 \mathrm{~Hz}$, and $\xi=0.22$. Since all amplifiers are alike, one transfer function is representative of each of their individual performance characteristics.

The transfer function $G_{\mathrm{a}}(s)$ can be converted into a statespace model as shown:

$$
\begin{aligned}
& \dot{x}_{a}=A_{a} x_{a}+B_{a} u_{a} \\
& y_{a}=C_{a} x_{a}
\end{aligned}
$$

Four eddy current displacement sensors are employed to measure the rotor lateral vibration in the $x$ and $y$ direction. The sensors are equipped with a low-pass filter to remove the carrier signal. According to the parameters provided by the sensor manufacturer, the integrated transfer function of the displacement sensors and the antialiasing filter $G_{s}(s)$ can be modeled as

$$
G_{s}(s)=\frac{K_{s}}{T_{s} s+1}
$$

where $K_{\mathrm{s}}=5000, T_{\mathrm{s}}=0.000056$.

3.4. Model Validation. The AMB is open-loop unstable, so system identification must be conducted in closed-loop condition. Figure 12 shows the scheme of the sweeping measurement. When the rotor is suspended at the equilibrium position under PID controller, the sine sweeping excitation signal is superimposed into the input end of the power amplifier (PA). The control signal superimposed with sine sweep and sensor signal is sent to the frequency response analyzer. In this way, the frequency response characteristic of control plant can be obtained as shown in Figure 13.

\section{Uncertainty Analysis Based on $v$-Gap Metric}

Rotor-AMB system has many uncertainties, such as complicated geometry, nonlinear material properties in magnetic bearing laminations, shrink fits, assembling, and manufacture error. These uncertainties must be considered during controller design. However, taking all the uncertainties into consideration in the controller design will result in large conservatism, which will decrease the controller performance and increase the controller order. Thus, the type of uncertainty should be classified according to its effect on closed-loop system robustness, determining which uncertainty can be easily suppressed by any feedback controller and 

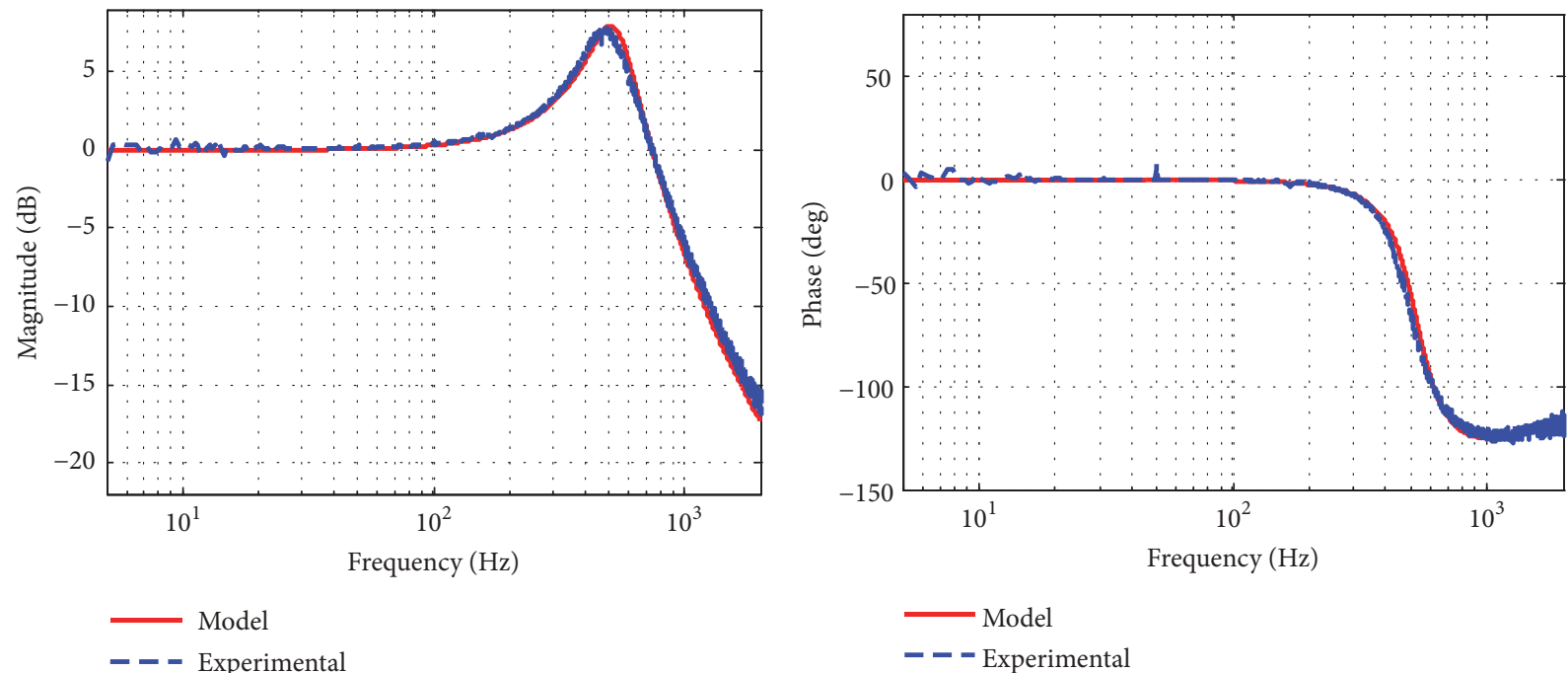

FIGURE 11: Frequency response of power amplifier.

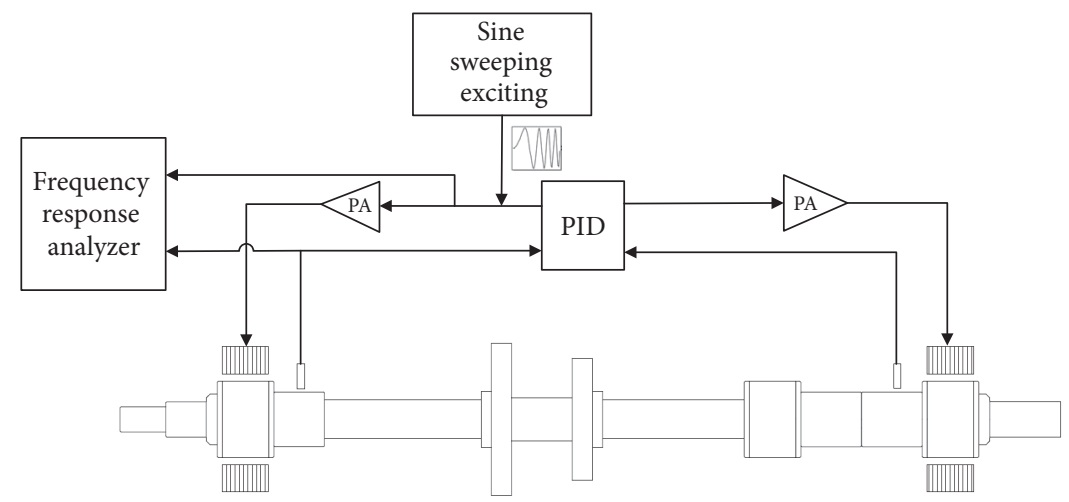

FIGURE 12: Scheme of sine sweeping measurement.

which uncertainty would have serious impact on closed-loop system stability. The uncertainty which has serious impact on closed-loop system stability must be explicitly addressed to reduce conservatism in the controller design.
In this work, $v$-gap metric is employed for uncertainty analysis because it can evaluate the effect of open-loop plant uncertainty from the closed-loop perspective without assuming a specific feedback controller [29].

The $v$-gap metric is defined in [30] as

$$
\delta_{v}\left(P_{1}, P_{2}\right)= \begin{cases}\left\|\widetilde{G}_{2} G_{1}\right\|_{\infty} & \text { if } \operatorname{det}\left(G_{2}^{*} G_{1}\right)(j \omega) \neq 0 \forall \omega \in(-\infty,+\infty) \text { and wno }\left[\operatorname{det}\left(G_{2}^{*} G_{1}\right)\right]=0 \\ 1 & \text { otherwise }\end{cases}
$$

where $G_{i}$ and $\widetilde{G}_{i}(i=1,2)$ are the corresponding normalized right and left coprime factorization of the plant $P_{1}$ and $P_{2}$; wno $(g)$ denotes the winding number about the origin of $g(s)$, as $s$ follows the standard Nyquist D-contour.

Nonconservative representation of uncertainty is the key to robust controller design. Based on the developed control plant model and physical system characteristics, some obvious uncertainties are extracted including loop gain, current stiffness $k_{i}$, displacement stiffness $k_{x}$, and rotor first bending mode frequency $\omega_{1}$. Uncertainty of $k_{i}$ and $k_{\mathrm{x}}$ can reach $15 \% \sim 20 \%$; especially $k_{x}$ have large changes due to the rotor displacement variation. Uncertainty of $\omega_{1}$ is usually set to $3 \% \sim 5 \%$ in the robust controller design. The loop gain uncertainty can reach $10 \%$. Table 2 shows the $v$-gap metric value of each uncertainty when they have maximum variation.

It is indicated from Table 2 that the $v$-gap metric of loop gain, $k_{i}$ and $k_{x}$, is small. These uncertainties can be well suppressed by any feedback controller. However, the $v$-gap metric value of rotor first bending frequency is very large even 

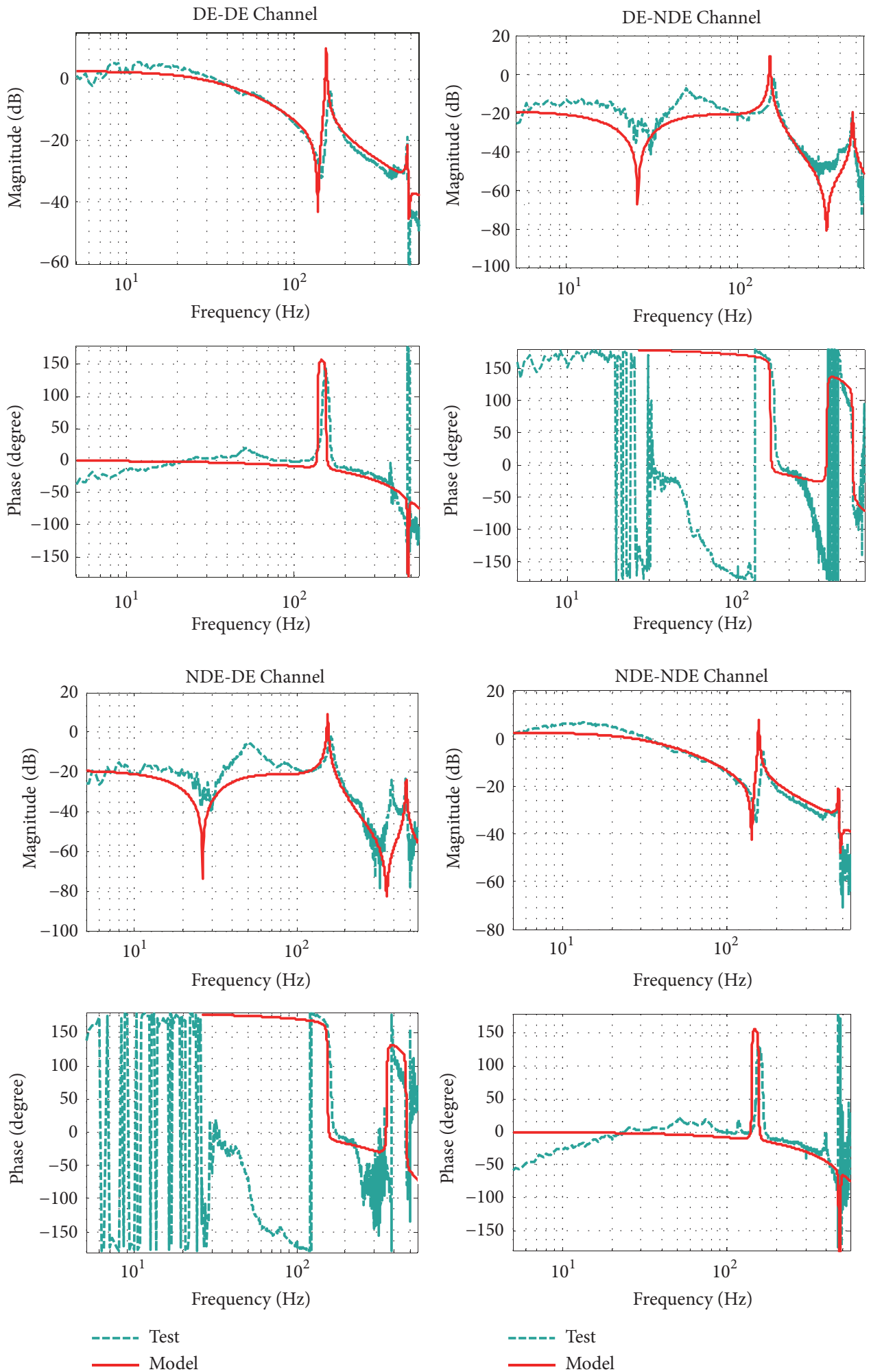

FIGURE 13: Frequency response of plant.

TABLE 2: v-gap metric value of uncertainties.

\begin{tabular}{lcccccccc}
\hline & \multicolumn{2}{c}{ loop gain } & \multicolumn{2}{c}{$k_{i}$} & & $k_{x}$ & $\omega_{1}$ \\
\hline variation & $-10 \%$ & $10 \%$ & $-15 \%$ & $15 \%$ & $-20 \%$ & $20 \%$ & $-5 \%$ & $5 \%$ \\
$v$-gap & 0.0526 & 0.0476 & 0.0811 & 0.0697 & 0.3703 & 0.3763 & 0.9835 & 0.9811 \\
\hline
\end{tabular}




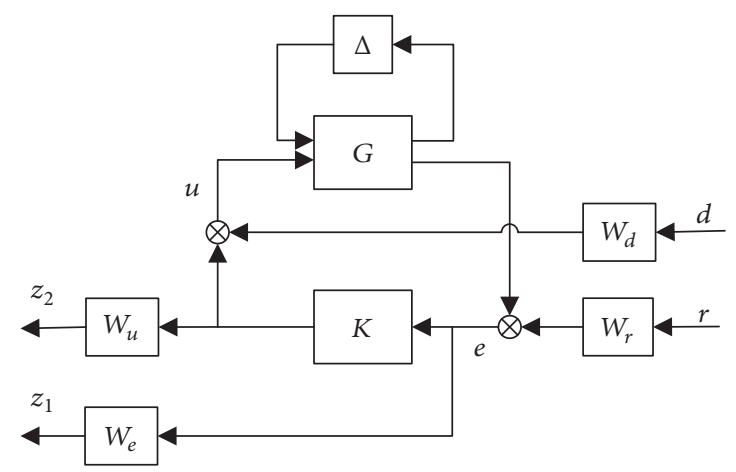

FIGURE 14: Block diagram of $\mu$-synthesis feedback loop.

if it has a small variation, which must be addressed explicitly [29].

Model error and changing operating condition always result in modal frequency uncertainty. Gyroscopic effect and shrink fit property due to speed and temperature variation will induce modal frequency variation. Therefore, it is very important to design AMB feedback systems that are robust to modal frequency uncertainty.

\section{5. $\mu$-Synthesis Controller Design}

For complex linear systems, $\mu$-synthesis is a multivariable controller design technique, which is highly suited for complex plants and MIMO control problems because it shows great superiority to handling flexible mode vibration. $\mu$ Synthesis takes the robust stability and performance as the optimized specifications explicitly and takes system uncertainties into account at the same time. These features are suitable for the flexible mode control of rotor-AMB system. Although $\mu$-synthesis is currently still rarely used, it shows high technical potential for industrial applications with high robustness to plant and other uncertainties. A detailed discussion of the advantage of $\mu$-synthesis for the control design of rotor-AMB system is given in [16].

We have made great modeling efforts for rotor-AMB system above and a relatively accurate system model was obtained. Based on v-gap, system uncertainties are analyzed. Special attention is paid to the design procedure of the modelbased $\mu$-synthesis controller in the following section. The block diagram of $\mu$-synthesis feedback loop design is shown in Figure 14, where $e$ is the tracking error, $u$ is the feedback control input, $d$ represents the external disturbance, $r$ is the reference signal, and $z_{1}$ and $z_{2}$ are weighted output. $\Delta$ is the parametric uncertainty, representing the rotor modal perturbation. $G$ is the physical system and $K$ is feedback controller which needs to be synthesized. $W_{u}, W_{e}, W_{d}$, and $W_{r}$ are weighting functions to capture the performance specifications.

The system gain from disturbance inputs to performance outputs is

$$
\left[\begin{array}{l}
z_{1} \\
z_{2}
\end{array}\right]=M\left[\begin{array}{l}
r \\
d
\end{array}\right]=\left[\begin{array}{cc}
W_{e} S W_{r} & W_{e} G S W_{d} \\
W_{u} K S W_{r} & W_{u} T W_{d}
\end{array}\right]\left[\begin{array}{l}
r \\
d
\end{array}\right]
$$

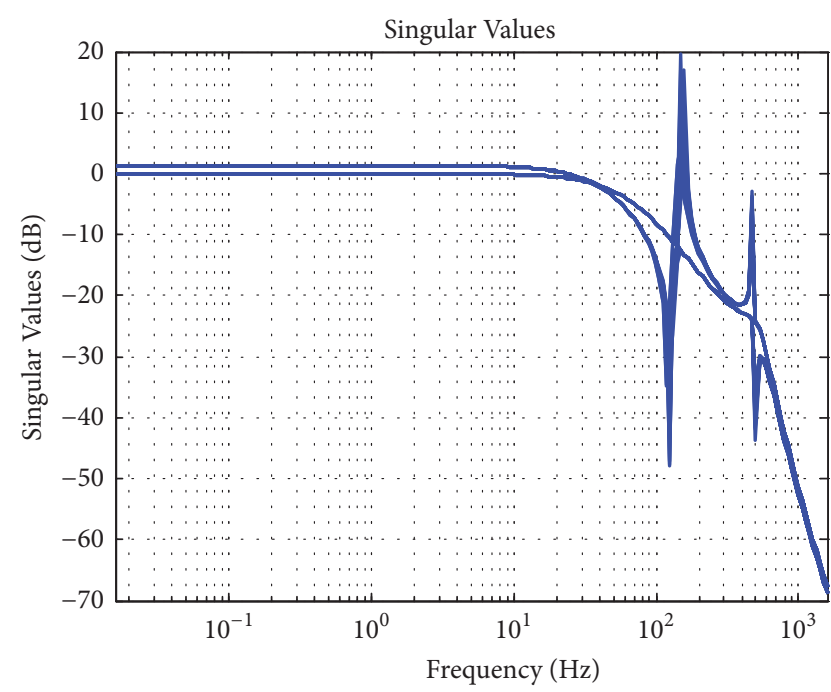

FIGURE 15: Singular value plot of uncertain system.

where $S$ is the system sensitivity function and $T$ is the complementary sensitivity function. The synthesis procedure is to find a controller $K$ making structured singular value

$$
\begin{aligned}
\mu_{\Delta}(M) & :=\frac{1}{\min \{\bar{\sigma}(\Delta): \Delta \in \Delta, \operatorname{det}(I-M \Delta)=0\}} \\
& <1
\end{aligned}
$$

5.1. Rotor Modal Frequency Uncertainty Representation. Appropriately accounting for model uncertainties is a crucial issue in the scope of robust control. The rotor modal frequency perturbation is parameter uncertainty with a very large $v$-gap metric value. System closed-loop robust stability is very sensitive to its variation. Hence, it should be addressed explicitly. In this paper, the real uncertainty representation is employed. The uncertain modal state matrix for the $i^{\text {th }}$ bending mode in a single plane can be expressed:

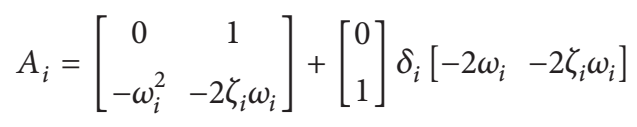

where $\omega_{i}$ is the bending mode frequency. $\zeta_{i}$ is the damping ratio of the mode. When the uncertainty $\delta_{i}$ is small, the quadratic term of $\delta_{i}$ can be omitted and the above result holds. The singular value plot of the uncertain plant dynamics is shown in Figure 15. The plot close to the first and second bending mode frequency has dense lines indicating the uncertainties we characterize.

5.2. Weighting Function Selection. The weighting function associated with the $\mu$-synthesis controller design can be difficult to calculate accurately and few formal techniques exist for the proper selection. Therefore, the selection of weighting functions can be directed based on the prior experience [10]. 
Generally, the weight $W_{r}$ is used to bound the system sensitivity peak. Thus, $W_{r}$ is usually chosen as a constant

$$
w_{r}=\frac{1}{M_{S}}
$$

where $M_{\mathrm{S}}=3$ represents the sensitivity peak according to ISO14839-3.

Weight $W_{d}$ is usually taken as a tune button to bound GS and is selected between $1 / 5$ and 1 . In this paper, weight $W_{d}$ is as

$$
w_{d}=0.6
$$

$W_{e}$ is the performance specification weight, which can strongly influence the steady error, overshoot and settling time of the rotor-AMB system. A constant $W_{e}$ weighting on sensitivity often results in a controller similar to a PD controller. In order to balance the rotor sag, a small integration is needed. Therefore,

$$
w_{e}=a \frac{s / M_{s}+\omega_{0}}{s+A \omega_{0}}
$$

where $\omega_{0}=30$ is the desired bandwidth and $A=0.0733$ is the integration constant; gain $a=2.6$ is used as additional parameter to help $W_{r}$ and $W_{d}$ bound the $S$ and GS.

In general, $W_{u}$ which bounds the control sensitivity function is often selected as second order high-pass filter, enforcing a sharper controller gain roll-off at high frequency. After much trial and error, the final $W_{u}$ is chosen as

$$
w_{u}=0.01 \cdot \frac{\omega_{1}^{2}}{\omega_{2}^{2}} \cdot \frac{s^{2}+0.707 \omega_{2} s+\omega_{2}^{2}}{s^{2}+0.707 \omega_{1} s+\omega_{1}^{2}}
$$

where $\omega_{2}=486$ represents the roll-off frequency and $\omega_{1}=$ $8 \omega_{2}$ add high frequency poles so that the inverse of the transfer function is proper.

Bode magnitude plots of the above weighting functions are shown in Figures 16 and 17. In general, these weighting functions are proper transfer functions.

The weighting functions defined above are SISO transfer function or scalar constants. However, in the controller synthesized procedure, the weights should be MIMO transfer function matrices. The performance requirement and dynamics of the four channels are similar. Therefore, the same weights are assigned to each channel.

$$
\begin{aligned}
& W_{e}=\operatorname{blkdiag}\left[w_{e}, w_{e}, w_{e}, w_{e}\right], \\
& W_{u}=\operatorname{blkdiag}\left[w_{u}, w_{u}, w_{u}, w_{u}\right] \\
& W_{r}=\operatorname{blkdiag}\left[w_{r}, w_{r}, w_{r}, w_{r}\right], \\
& W_{d}=\operatorname{blkdiag}\left[w_{d}, w_{d}, w_{d}, w_{d}\right]
\end{aligned}
$$

5.3. Synthesis Results and Evaluation of Controller. The Matlab function $d k s y n$ is used to perform the $D-K$ iteration steps of the $\mu$ controller synthesis. After several iterations, a 30th order controller is obtained. However, such a high order controller needs heavy calculation burden and is
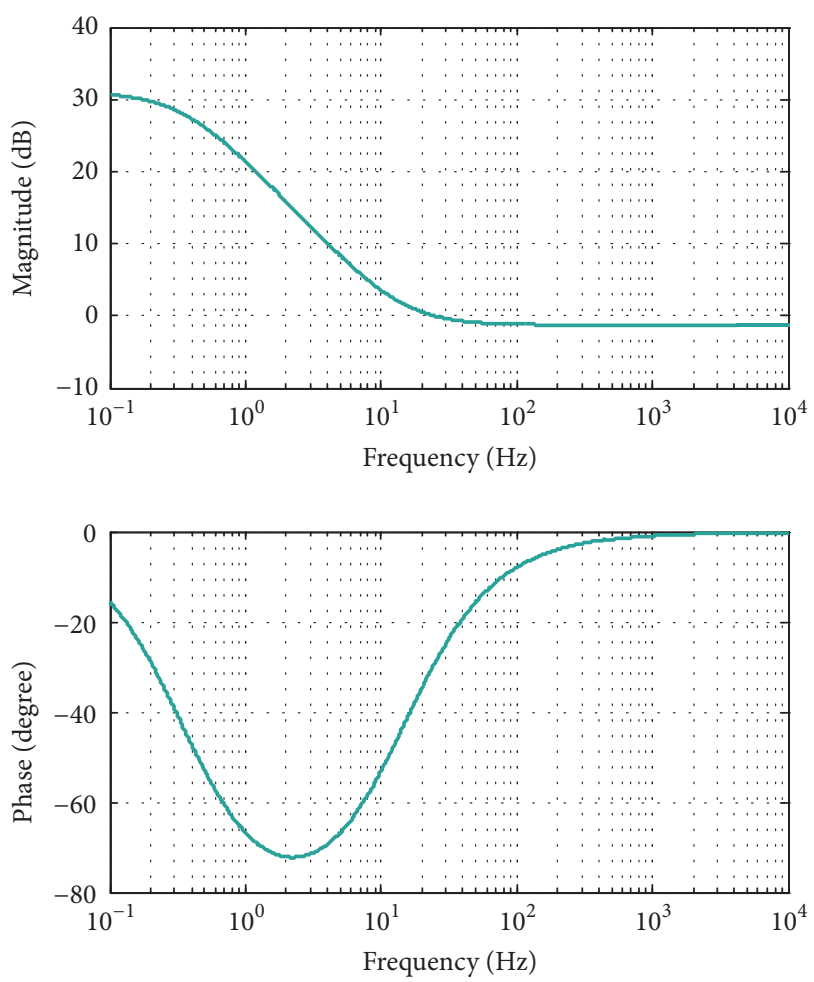

FIGURE 16: Weighting function of $w_{e}$.
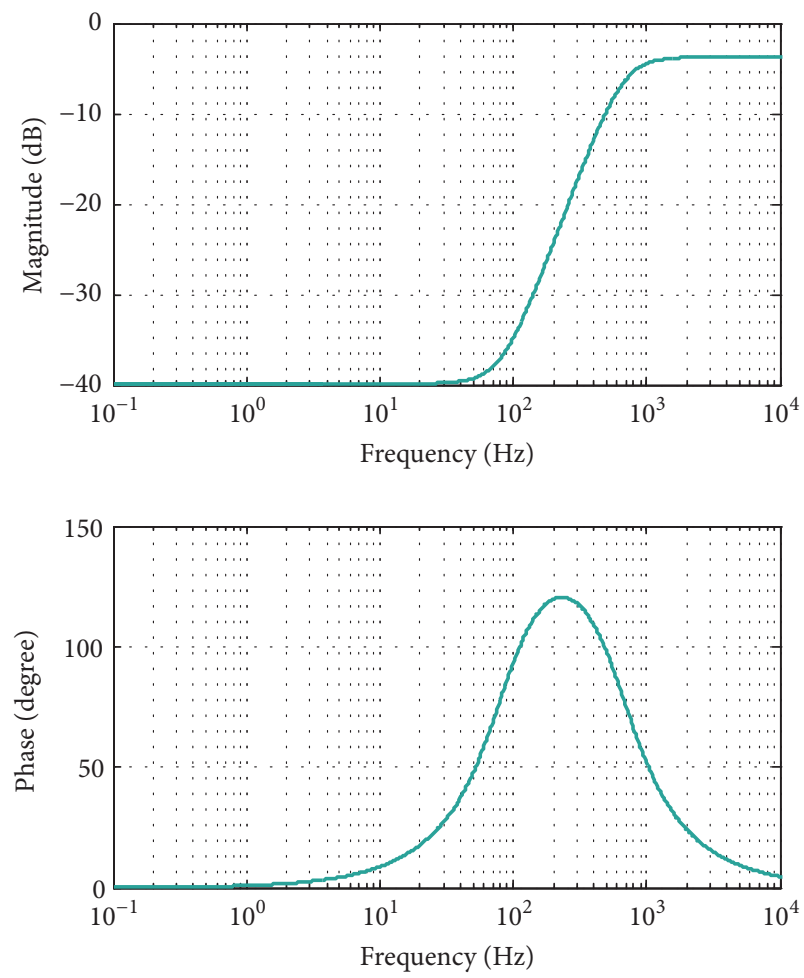

FIGURE 17: Weighting function of $w_{u}$.

difficult to implement with hardware limitations. Hence, an order-reduced controller with 14 states is retained based on 


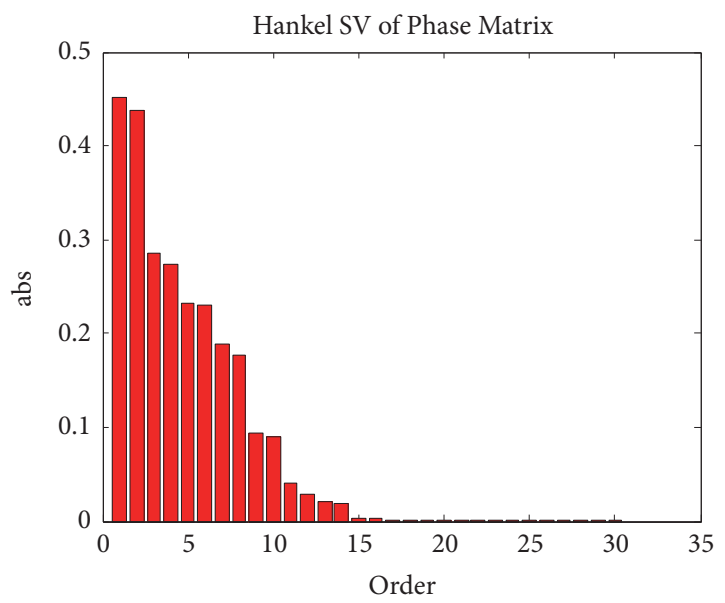

FIGURE 18: Hankel singular value of controller.

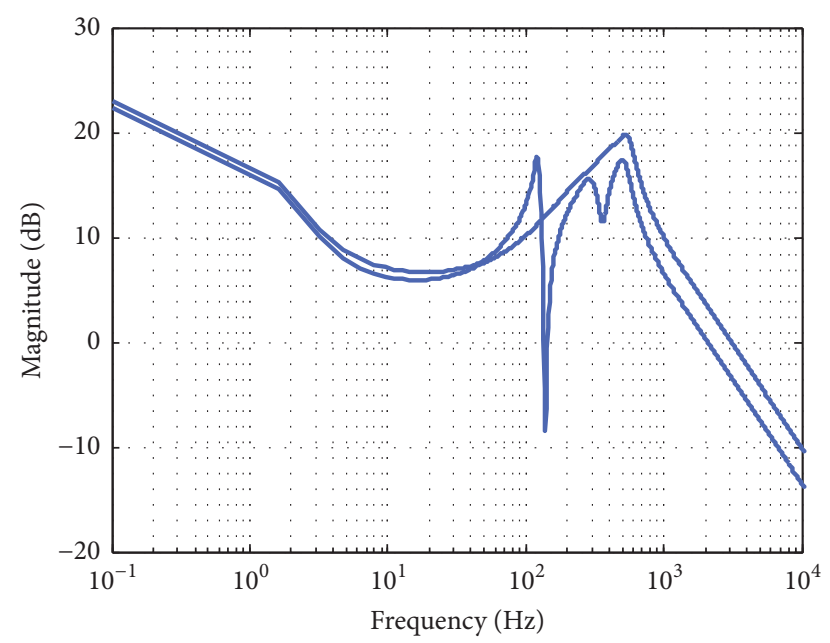

FIGURE 19: Singular value plot of u-synthesis controller.

balanced stochastic model truncation method. The Hankel singular values of the full-order controller are shown in Figure 18.

The singular value of the controller is shown in Figure 19. In the low frequency range, the controller has integral effect which is enforced by weighting function $W_{e}$. This can guarantee the rotor better steady error and static stiffness to balance rotor weight. In the middle frequency range, the controller is similar to the PD controller with stiffness and damping to stabilize the rigid body modes. In the high frequency, the controller places a series of poles and zeros to stabilize the flexible rotor bending modes and enforce sharp gain roll-off.

Sensitivity function is an important mean to evaluate the stability margin of the system [31]. Although the sensitivity peak is high in the rigid body mode, the low frequency uncertainty does not show a serious problem of robustness. On the contrary, the sensitivity in the flexible mode becomes more important. As can be seen from the $v$-gap value in the previous section, a slight perturbation at the flexible mode leads to serious closed-loop robust stability problems.

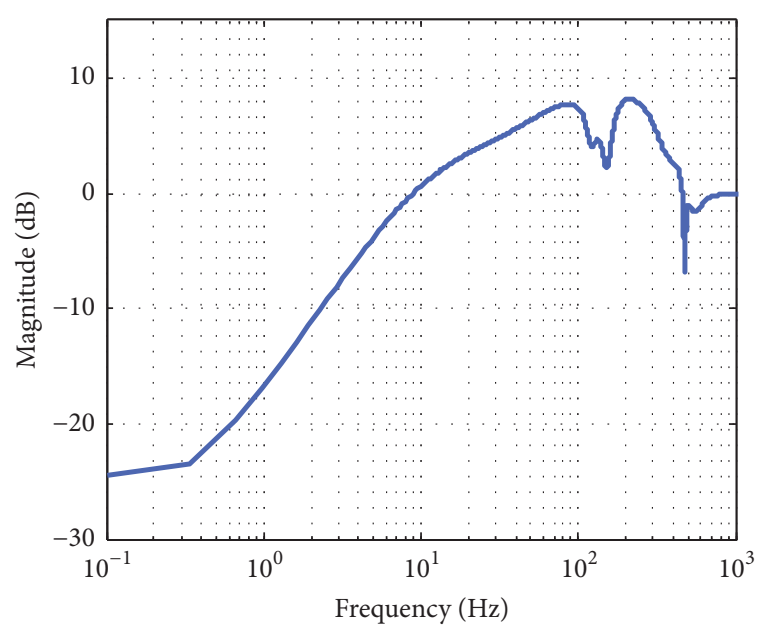

FIGURE 20: Sensitivity function of the DE-DE channel.

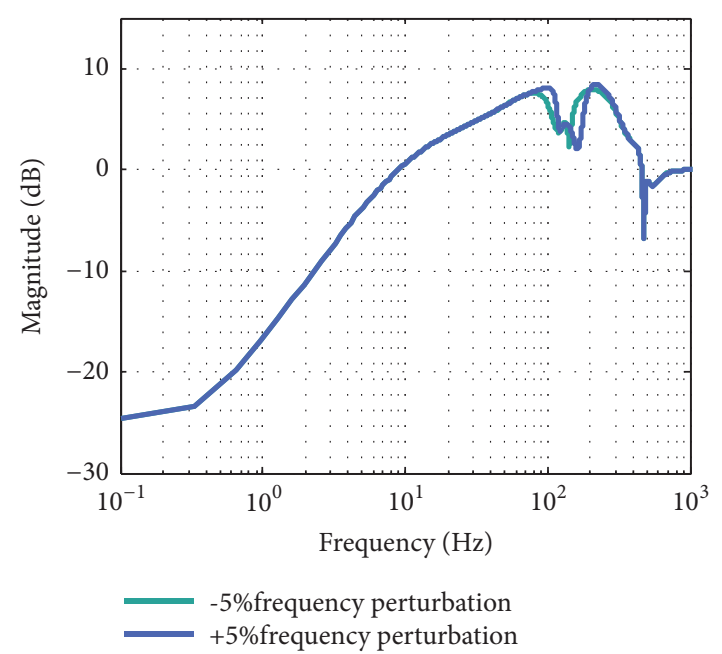

FIGURE 21: Sensitivity function with $\pm 5 \%$ natural frequency perturbation.

Hence, in order to verify the robustness of $\mu$-synthesis controller to rotor flexible mode perturbation, the output sensitivity functions of the DE-DE channel with $\pm 5 \%$ natural frequency perturbation are shown in Figures 20 and 21. It is indicated that system sensitivity at the flexible mode still has low bound with $\pm 5 \%$ natural frequency perturbation. The designed controller has high robustness to modal frequency uncertainty.

The system compliance transfer function is shown in Figure 22. In contrast to the sensitivity function measurement with the sole purpose of system robustness assessment, the compliance transfer function measurement constitutes a means for assessing the controlled system's performance, most notably in terms of damping of resonance [27]. From the compliance transfer function plot, the damping ratio of rotor first bending mode can be obtained with $17.35 \%$, which demonstrates the $\mu$-synthesis controller enforces much active damping for the flexible mode. 


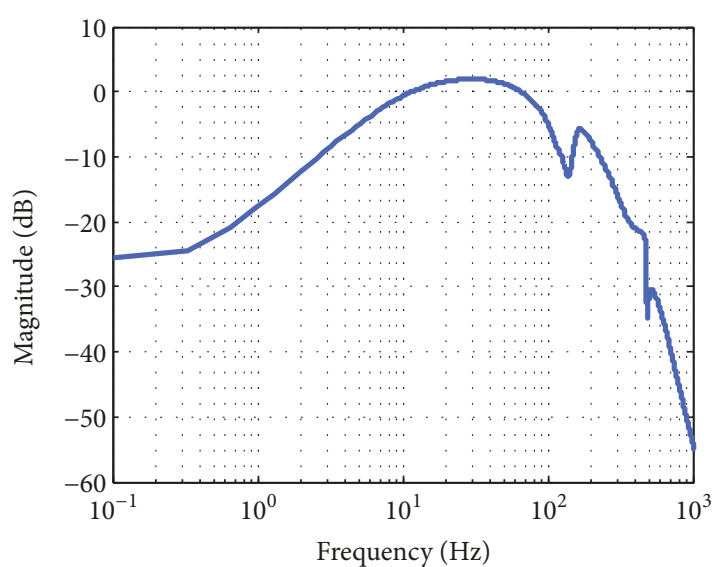

FIGURE 22: Compliance function of DE-DE channel.

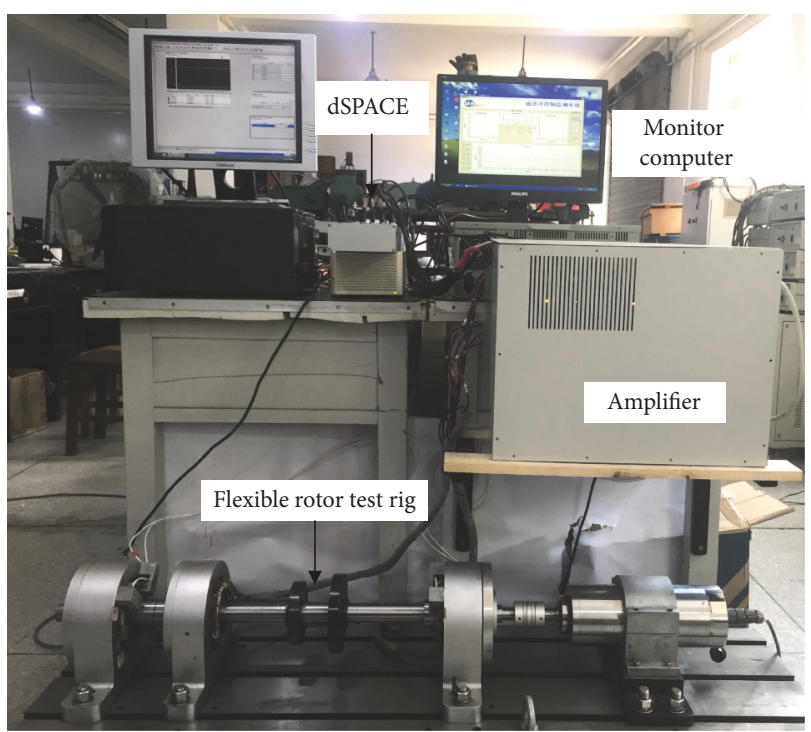

FIgURE 23: Photograph of the rotor-AMB test rig with major components.

\section{Experimental Validation}

The controller designed in Section 5 is implemented on the flexible rotor-AMB test rig. The experimental setup is shown in Figure 23, including dSPACE, monitor computer, amplifier, and flexible rotor test rig. The amplifier is a current feedback PWM switching amplifier. The PWM switching frequency is $26 \mathrm{kHz}$ and the amplifier bandwidth is $870 \mathrm{~Hz}$. The eddy current displacement sensor we used is MICROEPSILON made in Germany and its static resolution reaches $0.05 \mu \mathrm{m}$. A low-pass filter is connected in series after the displacement sensor to remove the high frequency noise. The $\mu$-synthesis controller is discretized at $20 \mathrm{kHz}$ and executed via the dSPACE DS1103 rapid control prototype. Detailed data of the experiment platform are listed in Table 3.

First, the initial levitation test is conducted with $\mu$ synthesis controller. Figure 24 shows the rotor positon from the rest state to the equilibrium position under $\mu$-synthesis
TABLE 3: Parameter of experiment platform.

\begin{tabular}{lc}
\hline Parameter & Value \\
\hline Rotor length & $0.93 \mathrm{~m}$ \\
Rotor mass & $18.14 \mathrm{~kg}$ \\
Rotor inertia moment & $0.016662 \mathrm{~kg} \cdot \mathrm{m}^{2}$ \\
Air gap & $0.5 \mathrm{~mm}$ \\
Displacement stiffness & $-1110.2 \mathrm{~N} / \mathrm{mm}$ \\
Current stiffness & $213.5 \mathrm{~N} / \mathrm{A}$ \\
Coil inductance & $8.8 \mathrm{mH}$ \\
Coil resistance & $1.2 \Omega$ \\
Coil turns & 192 \\
\hline
\end{tabular}

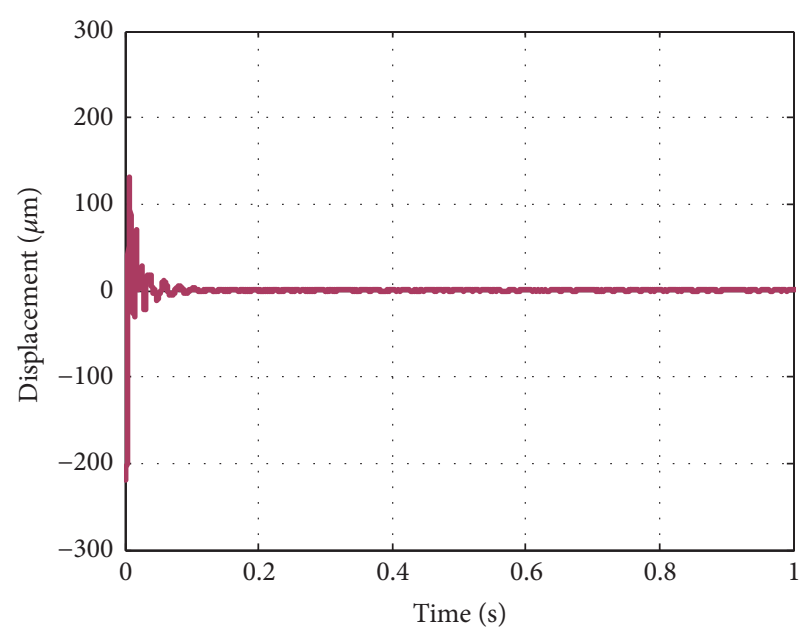

FIGURE 24: Levitation response under $\mu$ controller at the DE.

controller within $0.1 \mathrm{~s}$, which indicates good dynamic performance.

Sensitivity is an important specification of the robust stability margin. The sensitivity function can be measured directly by using online frequency sweep test [27]. Figure 25 shows the output sensitivity of the system under $\mu$-synthesis controller at the DE and NDE. It is indicated that the test results show good agreement with model prediction. The measured sensitivity peak is $9.405 \mathrm{~dB}$ located in Zone A [31]. The sensitivity nearby the rotor first bending mode under $\mu$-synthesis controller is much lower, which indicates that the $\mu$-synthesis controller has better robustness to the modal frequency uncertainty.

The compliance transfer functions at standstill are obtained by a swept sine measurement under $\mu$-synthesis controller. The nominal model was compared with the test data as shown in Figure 26. Based on the compliance measurement data, the damping ratios at the flexible mode can be estimated, which are $10.93 \%$ and $12.89 \%$, respectively, at the $\mathrm{DE}$ and NDE. The $\mu$ controller enforced much active damping on the first flexible mode.

The rotor run-up test is conducted. Figure 27 shows the rotor orbit changes with speed under $\mu$ controller. The flexible rotor successfully passes the first bending critical speed and reaches the rated speed $14400 \mathrm{rpm}$. The rotor orbit at the NDE shows obvious rigid mode critical speed peak. However, the 

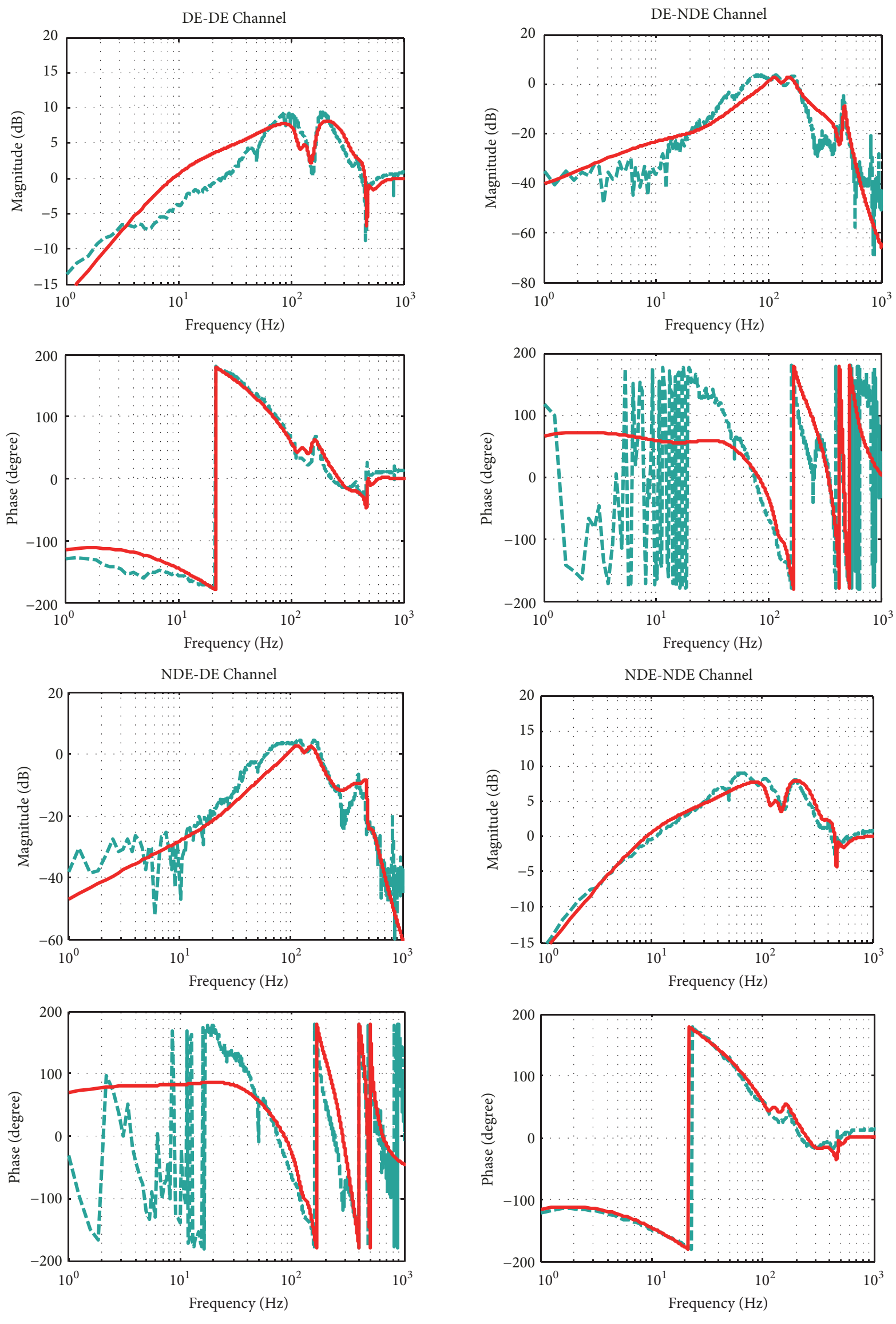

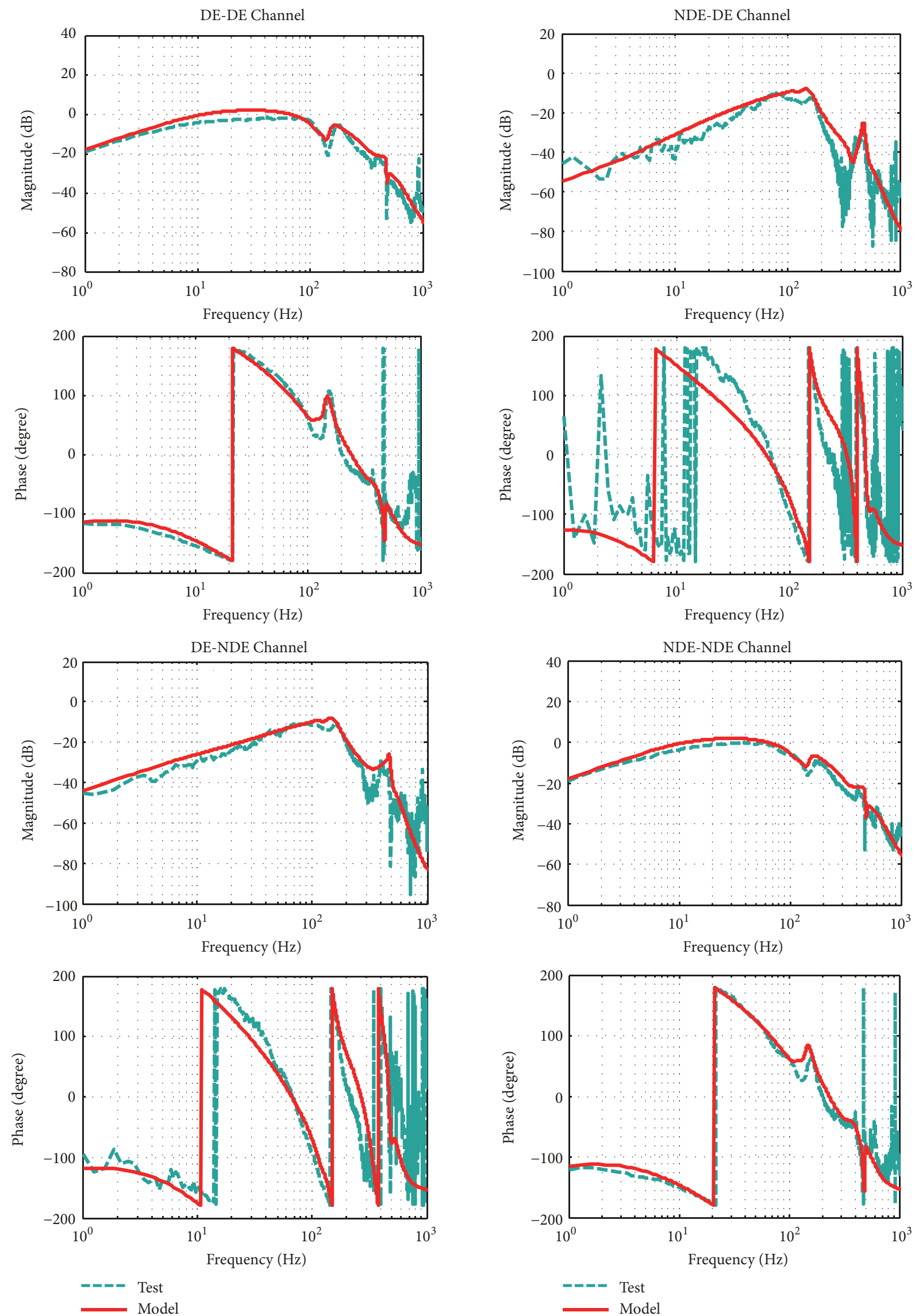

Figure 26: Compliance transfer function. 
Rotor orbit changes with speed at DE
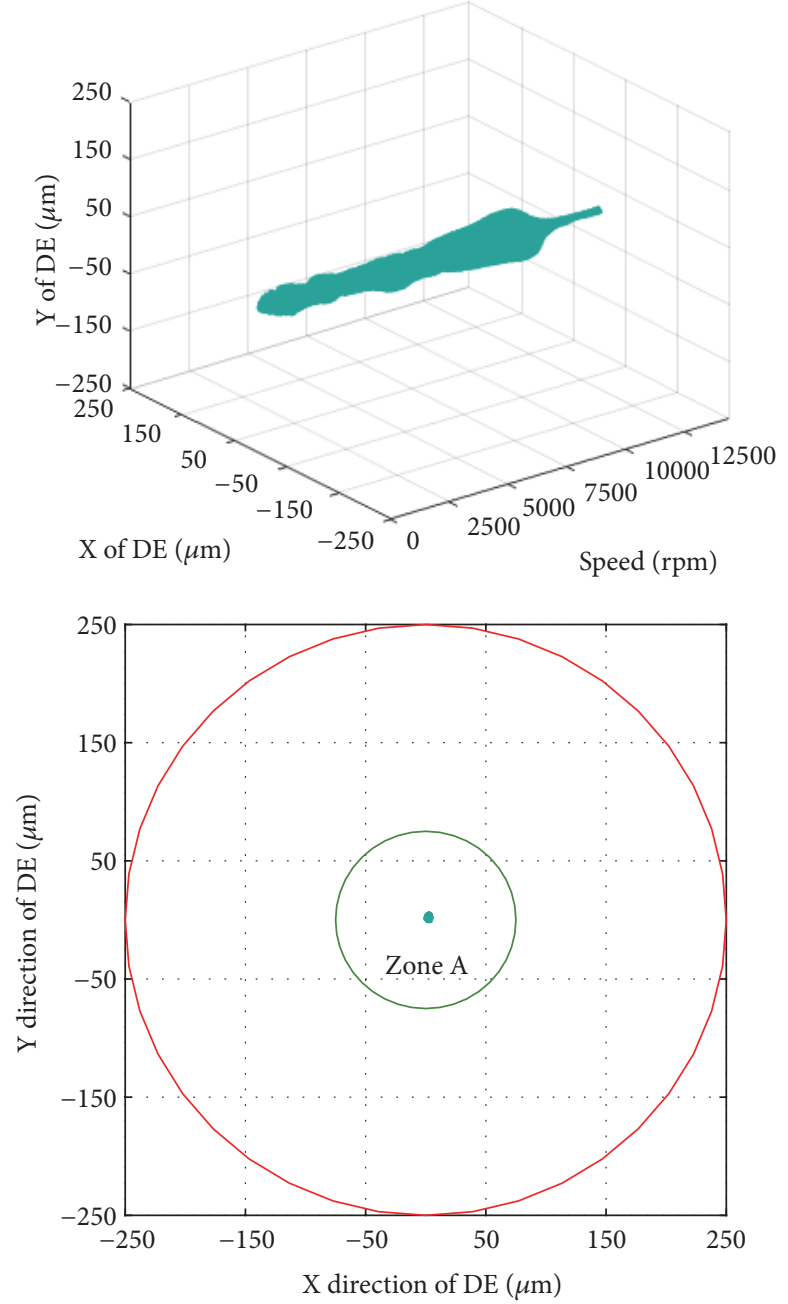

Rotor orbit changes with speed at NDE
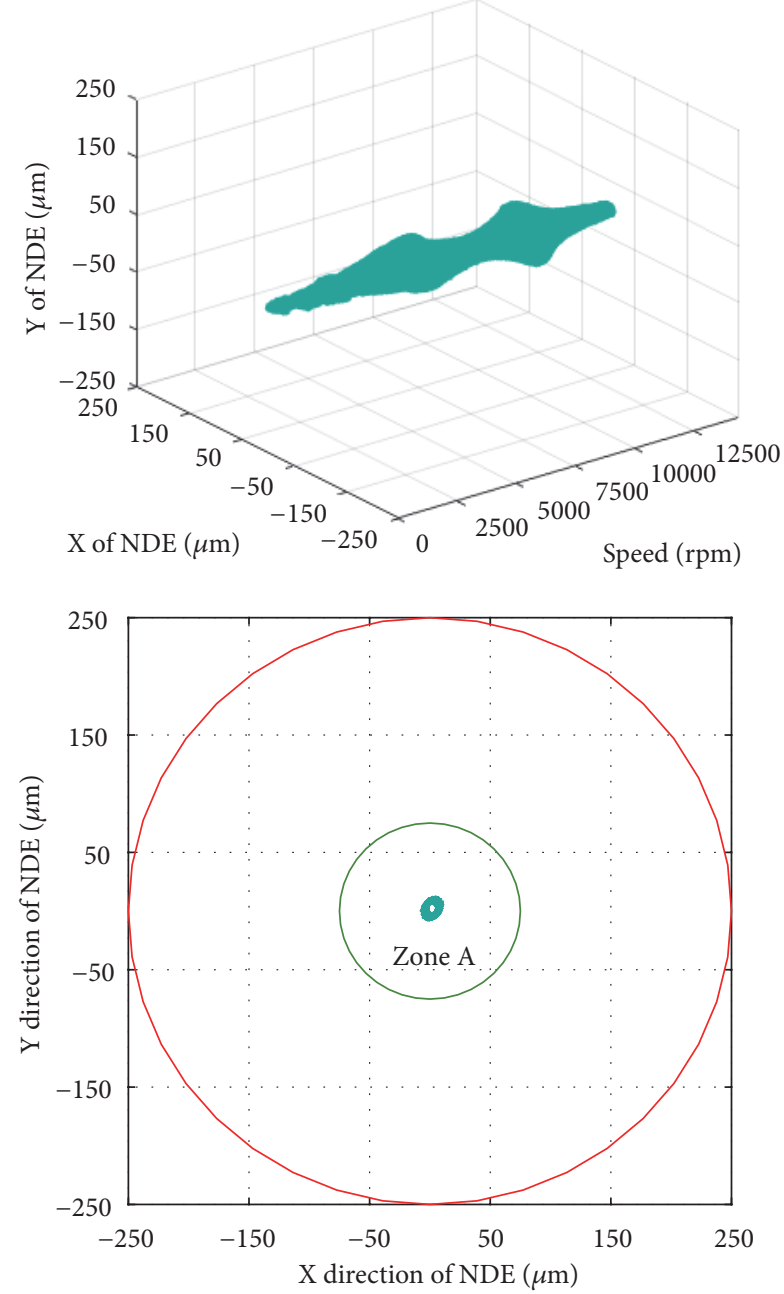

FIGURE 27: Rotor orbit.

orbit at the DE does not show obvious rigid mode critical speed peak due to the influence of flexible coupling. The rotor vibration level of steady-state operation at $14400 \mathrm{rpm}$ is located in Zone A [32].

\section{Conclusions}

In this paper, the resonance vibration control for $\mathrm{AMB}$ flexible rotor based on $\mu$-synthesis controller is investigated. A flexible rotor test rig is designed and the system model is obtained through the combination of system identification and theoretical modeling method. With the help of $v$-gap metric tool, rotor modal frequency perturbation has significant effect on the system stability, which must be addressed explicitly. The v-gap value reaches 0.9835 under $\pm 5 \%$ modal perturbation. Then, the $\mu$-synthesis controller is designed based on the uncertain system model. The simulation and experimental results show that the $\mu$ controller is robust to the modal perturbation and enforces much active damping on the first flexible mode (damping ratio reaches $12.89 \%$ ). In the run-up test, the rotor amplitude is within $45 \mu \mathrm{m}$ nearby the first bending critical speed, which indicates that the designed controller has good resonance vibration control performance for AMB flexible rotor system.

\section{Data Availability}

The data used to support the findings of this study are included within the article.

\section{Conflicts of Interest}

The authors declare that there are no conflicts of interest regarding the publication of this paper.

\section{Acknowledgments}

This research is financially supported by the National Natural Science Foundation of China (No. 51575411).

\section{References}

[1] H. Yefa, Z. Zude, and J. Zhengfeng, Basic Theory and Application of Magnetic Bearings, China Machine Press, 2006. 
[2] B. Han, Q. Xu, and Q. Yuan, "Multiobjective optimization of a combined radial-axial magnetic bearing for magnetically suspended compressor," IEEE Transactions on Industrial Electronics, vol. 63, no. 4, pp. 2284-2293, 2016.

[3] Y. Le and K. Wang, "Design and optimization method of magnetic bearing for high-speed motor considering eddy current effects," IEEE/ASME Transactions on Mechatronics, vol. 21, no. 4, pp. 2061-2072, 2016.

[4] C. Wang and J. Tang, "Design and mathematical analysis of a novel reluctance force-type hybrid magnetic bearing for flywheel with gimballing capability," Mathematical Problems in Engineering, vol. 2013, Article ID 836058, 17 pages, 2013.

[5] Q. Yuan, B. Han, and B. Dong, "The multiple objective optimization of high-speed rotor supported by magnetic bearing in BLDCM," International Journal of Applied Electromagnetics and Mechanics, vol. 46, no. 3, pp. 663-673, 2014.

[6] M. A. Rahman, A. Chiba, and T. Fukao, "Super high speed electrical machines - Summary," in Proceedings of the 2004 IEEE Power Engineering Society General Meeting, pp. 1272-1275, USA, June 2004.

[7] S. Islam, P. X. Liu, and A. El Saddik, "Robust control of fourrotor unmanned aerial vehicle with disturbance uncertainty," IEEE Transactions on Industrial Electronics, vol. 62, no. 3, pp. 1563-1571, 2015.

[8] C. Zhang, G. Zhao, and K. J. Tseng, "Robust decoupling control of a three-link robot arm directly driven by permanent magnet synchronous motors," International Journal of Applied Electromagnetics and Mechanics, vol. 36, no. 4, pp. 279-293, 2011.

[9] R. Conway, J. Choi, R. Nagamune, and R. Horowitz, "Robust track-following controller design in hard disk drives based on parameter dependent lyapunov functions," IEEE Transactions on Magnetics, vol. 46, no. 4, pp. 1060-1068, 2010.

[10] L. Guoxin, Robust stabilization of rotor-active magnetic bearing systems [Ph.D. thesis], University of Virginia, 2007.

[11] A. C. Wroblewski, Model identification, updating, and validation of an active magnetic bearing high-speed machining spindle for precision machining operation [Ph.D. Dissertation], Cleveland State University, 2011.

[12] T. Enqiong, F. Jiancheng, and H. Bangcheng, "Active vibration control of the flexible rotor in high energy density magnetically suspended motor with mode separation method," Journal of Engineering for Gas Turbines and Power, vol. 137, Article ID 082503, 2015.

[13] S. Zheng, H. Li, C. Peng, and Y. Wang, "Experimental investigations of resonance vibration control for noncollocated AMB flexible rotor systems," IEEE Transactions on Industrial Electronics, vol. 64, no. 3, pp. 2226-2235, 2017.

[14] E. Tang, B. Han, and Y. Zhang, "Optimum compensator design for the flexible rotor in magnetically suspended motor to pass the first bending critical speed," IEEE Transactions on Industrial Electronics, vol. 63, no. 1, pp. 343-354, 2016.

[15] Z. Gosiewski and A. Mystkowski, "The robust control of magnetic bearings for rotating machinery," Solid State Phenomena, vol. 113, pp. 125-130, 2006.

[16] E. H. Maslen and J. T. Sawicki, "Mu-synthesis for magnetic bearings: Why use such a complicated tool," ASME International Mechanical Engineering Congress and Exposition, pp. 1103-1112, 2007.

[17] A. Lanzon and P. Tsiotras, "Robust control of energy momentum wheels supported on active magnetic bearings using $\mathrm{H} \infty$ loop-shaping and $\mu$-synthesis," in Proceedings of the 15th World
Congress of the International Federation of Automatic Control, 2002, pp. 121-126, Spain, July 2002.

[18] H. Seto and T. Namerikawa, "An Hoo control system design of a magnetic bearing considering periodic disturbance," in Proceedings of the IEEE Conference on Control Applications, pp. 227-232, 2005.

[19] Z. Gosiewski and A. Mystkowski, "Robust control of active magnetic suspension: analytical and experimental results," Mechanical Systems and Signal Processing, vol. 22, no. 6, pp. 1297-1303, 2008.

[20] N. S. Gibson, H-infinity Control of Active Magnetic Bearings: An Intelligent Uncertainty Modeling Approach [Ph.D. Dissertation], North Carolina State University, 2004.

[21] S. J. M. Steyn, Multivariable Ho control for an active magnetic bearing flywheel system [M.S. Dissertation], North West University, 2010.

[22] A. Noshadi, J. Shi, W. S. Lee, P. Shi, and A. Kalam, "System identification and robust control of multi-input multi-output active magnetic bearing systems," IEEE Transactions on Control Systems Technology, vol. 24, no. 4, pp. 1227-1239, 2016.

[23] R. P. Jastrzebski, K. M. Hynynen, and A. Smirnov, "Ho control of active magnetic suspension," Mechanical Systems and Signal Processing, vol. 24, no. 4, pp. 995-1006, 2010.

[24] Y.-W. Long, Z.-Y. Xie, and X. Xu, "Ho robust control strategy for an active magnetic bearing," Journal of Vibration and Shock, vol. 32, no. 23, pp. 115-120, 2013.

[25] J. Zhou, S. Zheng, and B. Han, "Application of $\mu$ synthesis to the control of active magnetic bearings with parametric uncertainty," in Proceedings of the 20th International Conference on Electrical Machines and Systems (ICEMS), pp. 1-5, August 2017.

[26] S. E. Mushi, Z. Lin, and P. E. Allaire, "Stability analysis for a flexible rotor on active magnetic bearings subject to aerodynamic loads," in Proceedings of the 12th international symposium on magnetic bearings (ISMB '12), pp. 22-25, 2010.

[27] G. Schweitzer and E. H. Maslen, Magnetic Bearing: Theory, Design, and Application to Rotating Machinery, Springer, Berlin, Germany, 2009.

[28] M. I. Friswell, Dynamics of Rotating Machines, Cambridge University Press, Cambridge, UK, 2010.

[29] G. Li, Z. Lin, and P. Allaire, "Uncertainty classification for rotorAMB systems," in Proceedings of the International Symposium on Magnetic Bearings, 2006.

[30] G. Vinnicombe, "Uncertainty and feedback-Ho loop-shaping and the v-Gap metric," Uncertainty and feedback, pp. 117119, 2001.

[31] ISO 14839-3, Mechanical vibration - Vibration of rotating machinery equipped with active magnetic bearings - Part 3: Evaluation of stability margin, International Organization for Standardization ISO, 2006.

[32] ISO 14839-2, Mechanical vibration-Vibration of rotating machinery equipped with active magnetic bearings - Part 2: Evaluation of vibration, International Organization for Standardization ISO, 2006. 


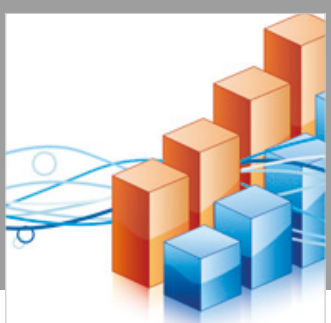

Advances in

Operations Research

\section{-n-m}
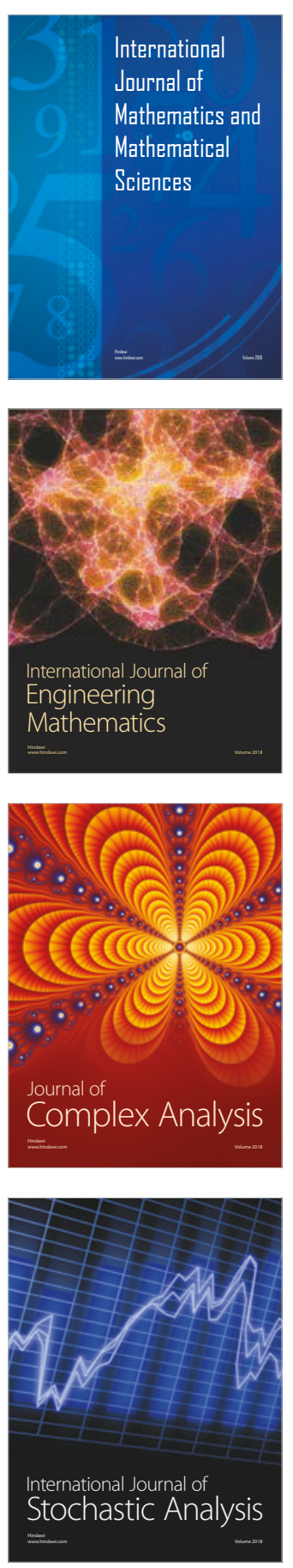
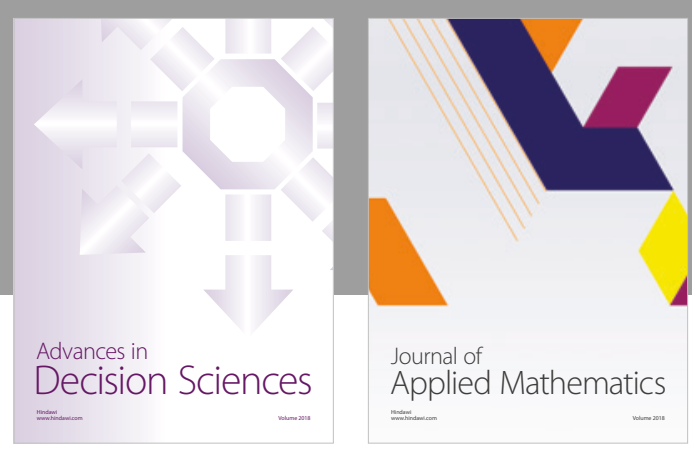

Journal of

Applied Mathematics
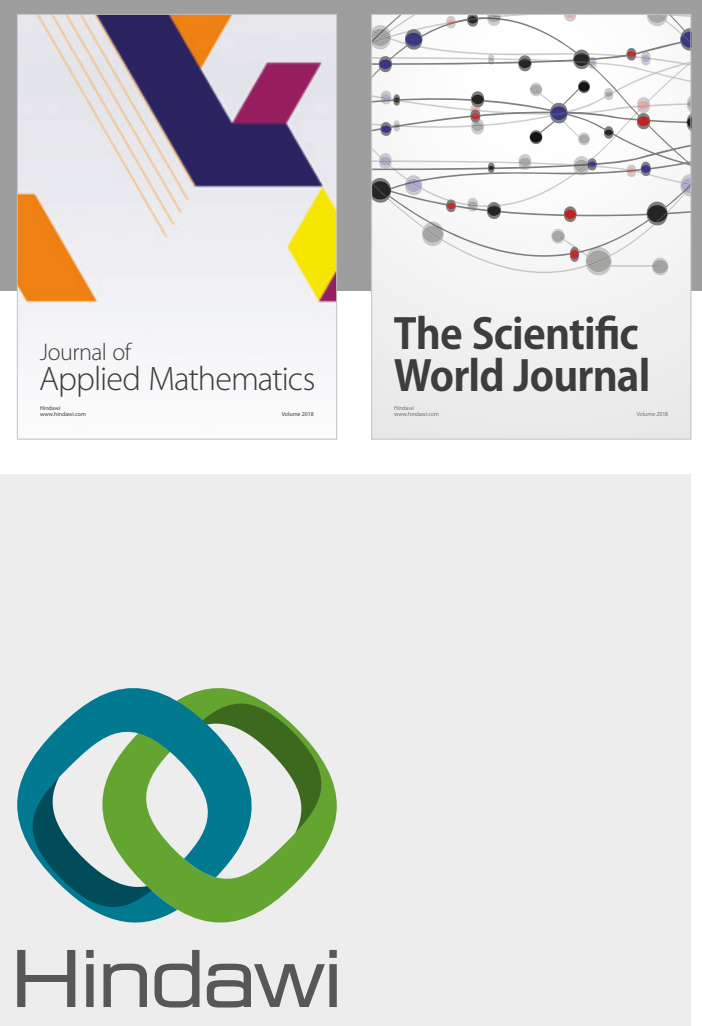

Submit your manuscripts at

www.hindawi.com

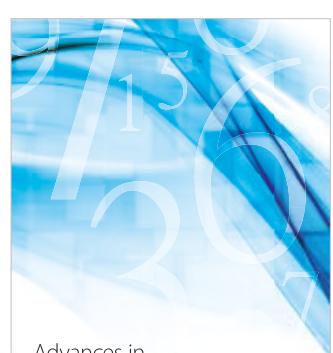

Advances in
Numerical Analysis
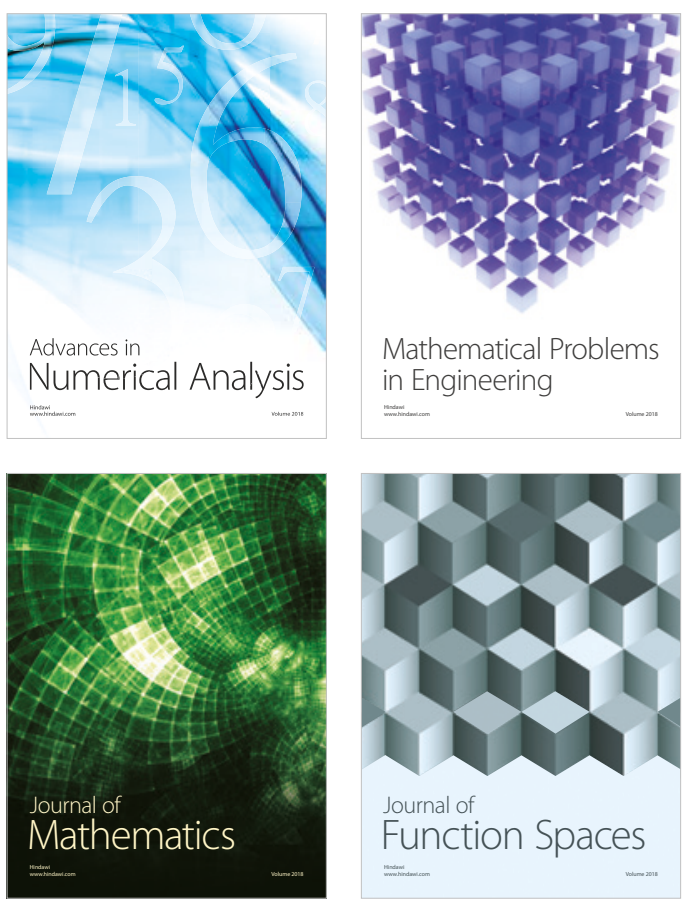

Mathematical Problems in Engineering

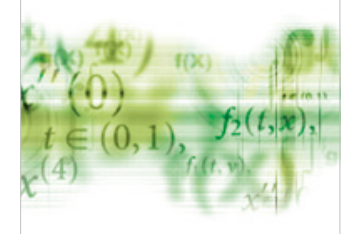

International Journal of

Differential Equations

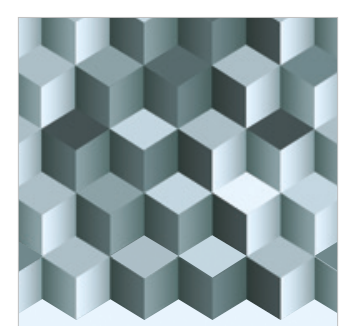

Journal of

Function Spaces

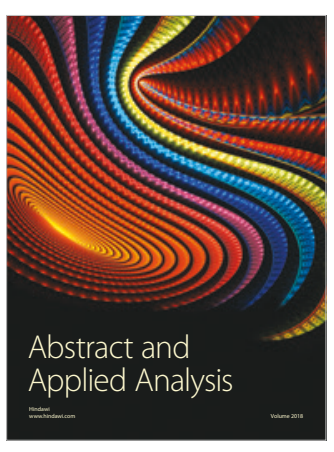

The Scientific

World Journal

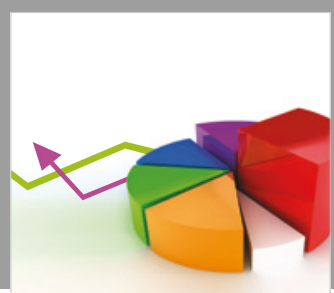

Journal of

Probability and Statistics
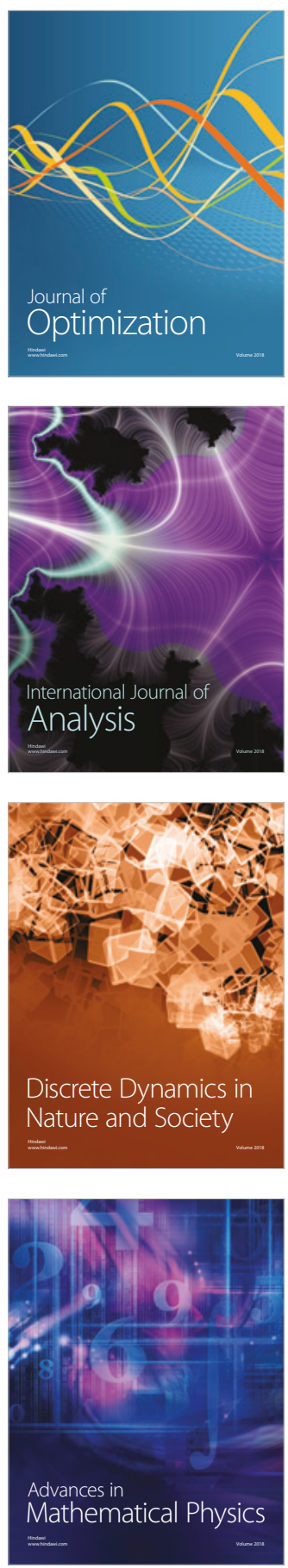